\title{
Not to normal order - Notes on the kinetic limit for weakly interacting quantum fluids
}

\author{
Jani Lukkarinen` and Herbert Spohn ${ }^{\dagger}$ \\ Department of Mathematics and Statistics, University of Helsinki, \\ P.O. Box 68, FI-00014 Helsingin yliopisto, Finland \\ Zentrum Mathematik, TU München, D-85747 Garching, Germany \\ * e-mail: jani.lukkarinen@ @elsinki.fi \\ † e-mail: spohn@ma.tum.de
}

Jürg Fröhlich and Tom Spencer have profoundly shaped the interface between physics and mathematics. It is therefore a particular pleasure to dedicate to them this article on a chapter of mathematical physics which is far from being closed.

\begin{abstract}
The derivation of the Nordheim-Boltzmann transport equation for weakly interacting quantum fluids is a longstanding problem in mathematical physics. Inspired by the method developed to handle classical dilute gases, a conventional approach is the use of the BBGKY hierarchy for the time-dependent reduced density matrices. In contrast, our contribution is motivated by the kinetic theory of the weakly nonlinear Schrödinger equation. The main observation is that the results obtained in the latter context carry over directly to weakly interacting quantum fluids provided one does not insist on normal order in the Duhamel expansion. We discuss the term by term convergence of the expansion and the equilibrium time correlation $\left\langle a(t)^{*} a(0)\right\rangle$.
\end{abstract}




\section{Introduction}

With the discovery of quantum mechanics, evidently, Boltzmann's kinetic theory of rarified gases had to be revised. The modification turned out to be minimal, in a certain sense. Only the classical differential scattering cross section had to be replaced by its quantum version, which thus depends on whether the gas particles are fermions or bosons. Otherwise the structure of the equation remains unaltered. In particular, the stationary solutions are still the Maxwellians. In addition, a natural task was to investigate the kinetic regime for weakly interacting quantum fluids with no particular restriction on the density. Timedependent perturbation theory, or even the more basic Fermi's golden rule, provides a convenient tool. Since the interaction is weak, the differential cross section appears now only in the Born approximation. The resulting quantum kinetic equation has a cubic collision operator, rather than a quadratic one as in Boltzmann's work. As a consequence, the Maxwellians $\exp [-\beta(\omega(k)-\mu)]$ are no longer stationary. The correct stationary solutions are of the form $(\exp [\beta(\omega(k)-\mu)] \mp 1)^{-1}$ in accordance with the statistics of the quantum fluid under consideration.

The quantum transport equation was first written down by Nordheim [1] in a paper submitted on May 30, 1928 and published a few weeks later. His work is an ingenious guess, supported by an H-theorem and by the physically expected stationary momentum distributions. Later on more systematic derivations followed [2]. In the kinetic literature the transport equation mostly carries the names of Uehling and Uhlenbeck [3]. In their 1933 paper they study properties of the transport equation, in particular its linearization around equilibrium and the long time hydrodynamic approximation.

There is a fundamental difference between weakly interacting classical and quantum fluids in the kinetic regime. For two classical particles a small interaction results in a small change of the relative momentum. Therefore the collision operator is not an integral operator, as in Boltzmann's classic work, but it is a nonlinear differential operator, apparently first realized in 1936 by Landau [4] in the context of plasmas and fluids with long range interactions. On the other hand, two weakly interacting quantum particles will pass through each other with probability $1-\mathcal{O}\left(\lambda^{2}\right), \lambda$ the coupling constant, and will s-wave scatter with probability $\mathcal{O}\left(\lambda^{2}\right)$. Therefore the collision operator is still an integral operator.

In our notes we will consider only quantum fluids interacting through a weak pair potential.

The conventional formal derivation of the transport equation proceeds via Fermi's golden rule. Over the years there have been many attempts to improve on the argument. On the theoretical side we mention in particular van Hove [5], Prigogine [6], and Hugenholtz [7]. With the work of Lanford [8] on the microscopic justification of the classical Boltzmann equation and the work of Davies [9] on the weak coupling limit for a small quantum system coupled to a large heat bath, the derivation of kinetic equations was recognized as a problem of interest to mathematical physics. In fact, it is already stated verbally in Hilbert's famous collection of problems as problem No. 6 [10]. The derivation of quantum kinetic equations still stands as a challenge. We will explain its current status 
in due course.

Regarding a quantum fluid as starting point, there are two in essence orthogonal ways to proceed with a semiclassical approximation. Within the particle picture, it is natural to take the limit of a large mass which leads to classical point particles interacting through a pair potential. On the other side, from the point of view of operator-valued fields, the natural limit is a weak, long range (on the scale of a typical inter-particle distance) potential which leads to the nonlinear Schrödinger equation, also known as Hartree equation. Both limits can be formulated as an Egorov theorem for operators [11, 12, 13, 14]. In the former case one is back to the model studied by Boltzmann while in the latter case the kinetic issue concerns a weakly nonlinear wave equation as already studied by Peierls [2].

In [15] we investigate the weakly nonlinear Schrödinger equation and develop a machinery for dealing with the high-dimensional oscillatory integrals as they arise in the Duhamel expansion of the solution of that equation. The goal of this paper is to explain how these novel techniques can be used for the derivation of quantum kinetic equations. In fact, we will provide mostly sketches of the argument and prove only a few crucial points. Otherwise we would be overwhelmed by technical issues. But we do clearly indicate where, to our understanding, there are gaps and new ideas will be required.

To give a brief outline: Weakly interacting lattice bosons and lattice fermions are introduced in Section 2 together with the Duhamel expansion of the time evolution operator. In Section 3 we explain how this expansion differs from the expansion resulting from the quantum BBGKY hierarchy and in Section 4 we make a comparison with wave turbulence for the weakly nonlinear Schrödinger equation. The main body of novel results are in Sections 5 to 7 where we discuss equilibrium time correlations and spatially homogeneous nonequilibrium states in the kinetic limit. In an appendix we summarize a few properties of spatially homogeneous quantum kinetic equations.

Acknowledgments. We would like to thank László Erdős for many illuminating discussions on the subject. The research was supported by a DFG grant and by the Academy of Finland. The work was partially done at the the Erwin Schrödinger Institute for Mathematical Physics (ESI), Vienna, Austria, whom we thank for their hospitality.

\section{Weakly interacting quantum fluids, Duhamel expan- sion}

We consider quantum particles on the $d$-dimensional lattice $\mathbb{Z}^{d}$ with lattice points $x \in \mathbb{Z}^{d}$. Later on there will be conditions which require $d \geq 3$. The particles "hop" independently to nearby sites. The hopping amplitude for the relative displacement is given by $\alpha$ : $\mathbb{Z}^{d} \rightarrow \mathbb{R}$ with $\alpha(x)=\alpha(-x)$. $\alpha$ is of compact support, i.e., $\alpha(x)=0$ for $|x|>R$ with suitable $R$. The Fourier transform of $\alpha$ is the dispersion relation $\omega(k)=\widehat{\alpha}(k)$. Clearly $\omega(k)=\omega(k)^{*}=\omega(-k)$ and $\omega$ is real analytic. This by itself will not be enough and further conditions, originating from the analysis in [15], will be imposed. The particles interact through the weak pair potential $\lambda V, V: \mathbb{Z}^{d} \rightarrow \mathbb{R}, V(x)=V(-x)$. $V$ is assumed 
to be of compact support and $\lambda>0, \lambda \ll 1$. Particles are either bosons or fermions. We introduce the corresponding annihilation/creation operators $a(x), a(x)^{*}, x \in \mathbb{Z}^{d}$. In the case of bosons they satisfy the commutation relations

$$
\left[a(x), a(y)^{*}\right]=\delta_{x y}, \quad[a(x), a(y)]=0, \quad\left[a(x)^{*}, a(y)^{*}\right]=0,
$$

while in the case of fermions they satisfy the anticommutation relations

$$
\left\{a(x), a(y)^{*}\right\}=\delta_{x y}, \quad\{a(x), a(y)\}=0, \quad\left\{a(x)^{*}, a(y)^{*}\right\}=0,
$$

$x, y \in \mathbb{Z}^{d}$. Here $[A, B]=A B-B A$ and $\{A, B\}=A B+B A$. With this notation the Hamiltonian of our system of particles reads

$$
H=\sum_{x, y \in \mathbb{Z}^{d}} \alpha(x-y) a(x)^{*} a(y)+\frac{1}{2} \lambda \sum_{x, y \in \mathbb{Z}^{d}} V(x-y) a(x)^{*} a(y)^{*} a(y) a(x) .
$$

$H$ at $\lambda=0$ is quadratic and denoted by $H_{\text {har }}$. Clearly the number of particles, $N$, is conserved,

$$
N=\sum_{x \in \mathbb{Z}^{d}} a(x)^{*} a(x) \quad \text { and } \quad[H, N]=0 .
$$

For $f: \mathbb{Z}^{d} \rightarrow \mathbb{C}$ we introduce its Fourier transform as

$$
\hat{f}(k)=\sum_{x \in \mathbb{Z}^{d}} f(x) \mathrm{e}^{-\mathrm{i} 2 \pi k \cdot x} .
$$

with $k \in \mathbb{T}^{d}=\left[-\frac{1}{2}, \frac{1}{2}\right]^{d}$, the $d$-dimensional unit torus. The physical momentum is $2 \pi k$, but our convention has the advantage of minimizing the number of $2 \pi$ prefactors. The inverse transform to (2.5) reads

$$
f(x)=\int_{\mathbb{T}^{d}} d k \hat{f}(k) \mathrm{e}^{\mathrm{i} 2 \pi k \cdot x} .
$$

Sometimes it is more convenient to have a function, say $W$, defined on $\mathbb{T}^{d}$. Then its inverse Fourier transform will be denoted by $\check{W}$. With this notation we have in momentum space

$$
\begin{gathered}
H=\int_{\mathbb{T}^{d}} d k \omega(k) \hat{a}(k)^{*} \hat{a}(k)+\frac{1}{2} \lambda \int_{\left(\mathbb{T}^{d}\right)^{4}} d k_{1} d k_{2} d k_{3} d k_{4} \delta\left(k_{1}+k_{2}-k_{3}-k_{4}\right) \\
\times \widehat{V}\left(k_{2}-k_{3}\right) \hat{a}\left(k_{1}\right)^{*} \hat{a}\left(k_{2}\right)^{*} \hat{a}\left(k_{3}\right) \hat{a}\left(k_{4}\right) .
\end{gathered}
$$

Remark 2.1 (Infinite volume limit). The Hamiltonians (2.3), resp. (2.7), define a unitary dynamics for initial states which have a bounded number of particles. To be more precise, we introduce the Fock space

$$
\mathfrak{F}=\bigoplus_{n=0}^{\infty} \mathfrak{F}_{n}, \quad \mathfrak{F}_{n}=\ell_{2}\left(\mathbb{Z}^{d}\right)^{\otimes n}
$$


and the projections $P_{\theta}, \theta= \pm . P_{+} \mathfrak{F}$ is the subspace of symmetric and $P_{-} \mathfrak{F}$ is the subspace of antisymmetric wave functions. Since $[H, N]=0, P_{\theta} \mathfrak{F}_{n}$ is invariant and $H$ restricted to $P_{\theta} \mathfrak{F}_{n}$ is a self-adjoint and bounded operator for each $n$. Of particular interest will be translation invariant initial states. Then the number of particles is infinite and the expression (2.3) is only formal. Thus one first has to restrict the fluid to a box $\Lambda$ with periodic boundary conditions. In the kinetic limit its side-length must be of order $\lambda^{-2}$. Thus, for constant density, the average number of particles is finite and of order $\lambda^{-2 d}$. Hence, for fixed $\lambda$ the dynamics is well defined. Conceptually one would prefer to first let $\Lambda \uparrow \mathbb{Z}^{d}$ and then $\lambda \rightarrow 0$ together with the appropriate rescaling of space-time. This program can be carried through for fermions, in which case the operators $a(x), x \in$ $\mathbb{Z}^{d}$, are bounded and the dynamics is defined as a group of automorphisms on the $C^{*}$ algebra of quasi-local observables [16]. Thus one can work directly at infinite volume. For lattice bosons the dynamics at infinite volume is not so well understood. For our purposes the infinite volume limit is not a central issue. One can first write down the Duhamel expansion in finite volume $\Lambda$ and then establish that, for the particular initial state and the observables of interest, the estimates are uniform in $\Lambda$, hence hold when $\Lambda \uparrow \mathbb{Z}^{d}$.

Remark 2.2 (Stability). We will study equilibrium time correlations in the kinetic limit. For this the $\beta$-KMS state $\langle\cdot\rangle_{\beta, \lambda}$ for $H-\mu N, \mu$ the chemical potential, has to be welldefined and, hence, $V$ has to be a thermodynamically stable potential. For lattice fermions no further conditions are needed. For lattice bosons a simple sufficient condition would be

$$
V \geq 0
$$

For a discussion of equilibrium states at small $\lambda$ we refer to [17, 18]. In the kinetic limit thermodynamic stability does seem to play a role.

Remark 2.3 (Continuum limit). In [15] we study a classical field theory by regarding $a(x), a(x)^{*}$ as commutative complex-valued field. To avoid ultraviolet divergence, it was necessary to introduce a spatial discretization, as will be explained in more detail in Section 4. Since we plan to transcribe the results from [15] to quantum fluids, we stick to the lattice theory. Thus our model is appropriate, for example, for a fluid of electrons in a crystal background potential and for bosons in an optical lattice. To understand whether a continuum limit is feasible, we recall that

$$
\left\langle\hat{a}(k)^{*} \hat{a}\left(k^{\prime}\right)\right\rangle_{\beta, 0}=\delta\left(k-k^{\prime}\right) W_{\beta}^{ \pm}(k), \quad W_{\beta}^{ \pm}(k)=\frac{1}{\mathrm{e}^{\beta(\omega(k)-\mu)} \mp 1} .
$$

Here $\beta$ is the inverse temperature, $\beta>0$, and $\mu$ is the chemical potential chosen such that $\omega(k)-\mu>0$ (and suppressed in our notation). We adopt the standard convention $W_{\beta}^{\theta}$, where $\theta=1$ stands for bosons and $\theta=-1$ for fermions.

In the continuum limit, i.e., for a fluid in empty space, one would replace the position space $\mathbb{Z}^{d}$ by $\mathbb{R}^{d}$ and $\alpha *$ by $-\Delta$, implying the dispersion relation $\omega(k)=k^{2}$. Clearly in this case

$$
\int_{\mathbb{R}^{d}} W_{\beta}^{ \pm}(k) d k<\infty
$$


indicating that there is no ultraviolet divergence. Indeed, as discussed in [17, 18], the equilibrium state for small $\lambda$, and with restrictions on the density, is well-defined. It would be of considerable interest to find out whether the analysis in [15] could be extended to weakly interacting quantum fluids in $\mathbb{R}^{d}$.

For an arbitrary operator $A$ we define its Heisenberg evolution by $A(t)=\mathrm{e}^{\mathrm{i} H t} A \mathrm{e}^{-\mathrm{i} H t}$, $A(0)=A$. Using $\frac{d}{d t} A(t)=\mathrm{i}[H, A(t)]$, one obtains the evolution equation

$$
\frac{d}{d t} a(x, t)=-\mathrm{i} \sum_{y \in \mathbb{Z}^{d}} \alpha(x-y) a(y, t)-\mathrm{i} \lambda \sum_{y \in \mathbb{Z}^{d}} V(x-y) a(y, t)^{*} a(y, t) a(x, t),
$$

which in momentum space becomes

$$
\begin{aligned}
& \frac{d}{d t} \hat{a}\left(k_{1}, t\right)=-\mathrm{i} \omega\left(k_{1}\right) \hat{a}\left(k_{1}, t\right)-\mathrm{i} \lambda \int_{\left(\mathbb{T}^{d}\right)^{3}} d k_{2} d k_{3} d k_{4} \delta\left(k_{1}+k_{2}-k_{3}-k_{4}\right) \\
& \times \widehat{V}\left(k_{2}-k_{3}\right) \hat{a}\left(k_{2}, t\right)^{*} \hat{a}\left(k_{3}, t\right) \hat{a}\left(k_{4}, t\right)
\end{aligned}
$$

Equations (2.12) and (2.13) will be solved as a Cauchy problem given the initial, time $t=0$, state. In general one should allow for spatial variation on the scale $\lambda^{-2}$. To keep matters simple we will restrict ourselves to a spatially homogeneous situation. Thus the initial state $\langle\cdot\rangle$ is assumed to be translation invariant with good clustering properties. In particular

$$
\left\langle\hat{a}(k)^{*} \hat{a}\left(k^{\prime}\right)\right\rangle=\delta\left(k-k^{\prime}\right) W(k)
$$

for some smooth $W$. In addition, $\langle\cdot\rangle$ has to be gauge invariant, i.e.,

$$
\left\langle\mathrm{e}^{\mathrm{i} \vartheta N} A \mathrm{e}^{-\mathrm{i} \vartheta N}\right\rangle=\langle A\rangle
$$

for $\vartheta \in[0,2 \pi]$. One quantity of interest will be the two-point function at time $t$. Since the dynamics preserves translation and gauge invariance, it must necessarily be of the form

$$
\left\langle\hat{a}(k, t)^{*} \hat{a}\left(k^{\prime}, t\right)\right\rangle=\delta\left(k-k^{\prime}\right) W_{\lambda}(k, t)
$$

$W_{\lambda} \geq 0, W_{\lambda}(k, 0)=W(k)$, together with $\langle\hat{a}(k, t)\rangle=0,\left\langle\hat{a}(k, t) \hat{a}\left(k^{\prime}, t\right)\right\rangle=0$.

Kinetic theory studies $W_{\lambda}\left(k, t / \lambda^{2}\right)$ for small $\lambda$. A special role will be played by quasifree states, which we define first.

Definition 2.4 (quasifree state). Let $\rho_{1}$ be a self-adjoint operator on $\ell_{2}\left(\mathbb{Z}^{d}\right)$ with integral kernel $\rho_{1}(x, y)$. For bosons we impose $\rho_{1} \geq 0$ while for fermions $0 \leq \rho_{1} \leq 1$. A gauge invariant state $\langle\cdot\rangle$ is called quasifree with correlator $\rho_{1}$, if for all $x_{i}, y_{j} \in \mathbb{Z}^{d}$, $i=1, \ldots, m, j=1, \ldots, n$, it holds

$$
\left\langle\left(\prod_{i=1}^{m} a\left(x_{i}\right)\right)^{*}\left(\prod_{j=1}^{n} a\left(y_{j}\right)\right)\right\rangle=\delta_{m n} \operatorname{det}_{\theta}\left(\rho_{1}\left(x_{i}, y_{j}\right)\right)_{i, j=1, \ldots, n} .
$$


Here the operators are ordered from left to right as they appear in the $\prod$ symbol and the empty product is interpreted as 1 . det - is the usual determinant, while det $t_{+}$is the permanent. Translation invariance is reflected by $\rho_{1}(x, y)$ depending only on $x-y$, resp. in momentum space by (2.14).

Kinetic theory puts forward a rather simple picture of the dynamics for small $\lambda$ : On the microscopic time scale the initial $W(k)$ does not change while the unperturbed dynamics forces the state to become quasifree. To say, after a time $t$ which is short on the kinetic scale and long on the microscopic scale, for arbitrary $n \in \mathbb{N}$, the higher moments are approximately of the form, $\check{W}$ denoting the inverse Fourier transform,

$$
\left\langle\left(\prod_{i=1}^{n} a\left(x_{i}, t\right)\right)^{*}\left(\prod_{j=1}^{n} a\left(y_{j}, t\right)\right)\right\rangle \simeq \operatorname{det}_{\theta}\left(\check{W}\left(x_{i}-y_{j}\right)\right)_{i, j=1, \ldots, n}
$$

with all other moments vanishing. On the kinetic time scale, $t=\mathcal{O}\left(\lambda^{-2}\right), W_{\lambda}(k, t)$ changes while preserving the quasifree property. Of course, only in the limit $\lambda \rightarrow 0$ we obtain such a strict separation of the two space-time scales. The initial time slip with the dynamics generated by $H_{\text {har }}$ was studied by Ho and Landau [19]. Under suitable assumptions on $\omega$, they prove quasifreeness in the limit $t \rightarrow \infty$ provided the initial state is $\ell_{1}$-clustering.

We are mostly interested in the kinetic time scale and thus impose the state $\langle\cdot\rangle$ to be quasifree to begin with. As argued in [20], see also [21], if one assumes, up to small errors, the state still to be quasifree at the long time $t=\lambda^{-2} \tau, \tau=\mathcal{O}(1)$, then it is not too difficult to determine the (approximate) evolution equation for $W_{\lambda}(k, t)$.

To study $\left\langle\hat{a}(k, t)^{*} \hat{a}\left(k^{\prime}, t\right)\right\rangle$ the only method currently available is to expand the expectation value of interest with respect to $\lambda$, which is achieved through the Duhamel expansion. It will be convenient to work in the interaction representation and we introduce

$$
\hat{a}(k, 1, t)=\mathrm{e}^{\mathrm{i} \omega(k) t} \hat{a}(k, t), \quad \hat{a}(k,-1, t)=\mathrm{e}^{-\mathrm{i} \omega(k) t} \hat{a}(-k, t)^{*} .
$$

Then (2.13) becomes

$$
\begin{aligned}
\frac{d}{d t} \hat{a}\left(k_{1}, \sigma, t\right) & =-\mathrm{i} \lambda \sigma \int_{\left(\mathbb{T}^{d}\right)^{3}} d k_{2} d k_{3} d k_{4} \delta\left(k_{1}-k_{2}-k_{3}-k_{4}\right) \\
& \times \frac{1}{2}\left((1+\sigma) \widehat{V}\left(k_{2}+k_{3}\right)+(1-\sigma) \widehat{V}\left(k_{3}+k_{4}\right)\right) \\
& \times \exp \left[-\mathrm{i} t\left(-\sigma \omega\left(k_{1}\right)-\omega\left(k_{2}\right)+\sigma \omega\left(k_{3}\right)+\omega\left(k_{4}\right)\right)\right] \\
& \times \hat{a}\left(k_{2},-1, t\right) \hat{a}\left(k_{3}, \sigma, t\right) \hat{a}\left(k_{4}, 1, t\right) .
\end{aligned}
$$

For a product it holds that

$$
\frac{d}{d t} \prod_{j=1}^{n} \hat{a}\left(k_{j}, \sigma_{j}, t\right)=\sum_{m=1}^{n}\left(\prod_{j=1}^{m-1} \hat{a}\left(k_{j}, \sigma_{j}, t\right)\right) \frac{d}{d t} \hat{a}\left(k_{m}, \sigma_{m}, t\right)\left(\prod_{j=m+1}^{n} \hat{a}\left(k_{j}, \sigma_{j}, t\right)\right)
$$




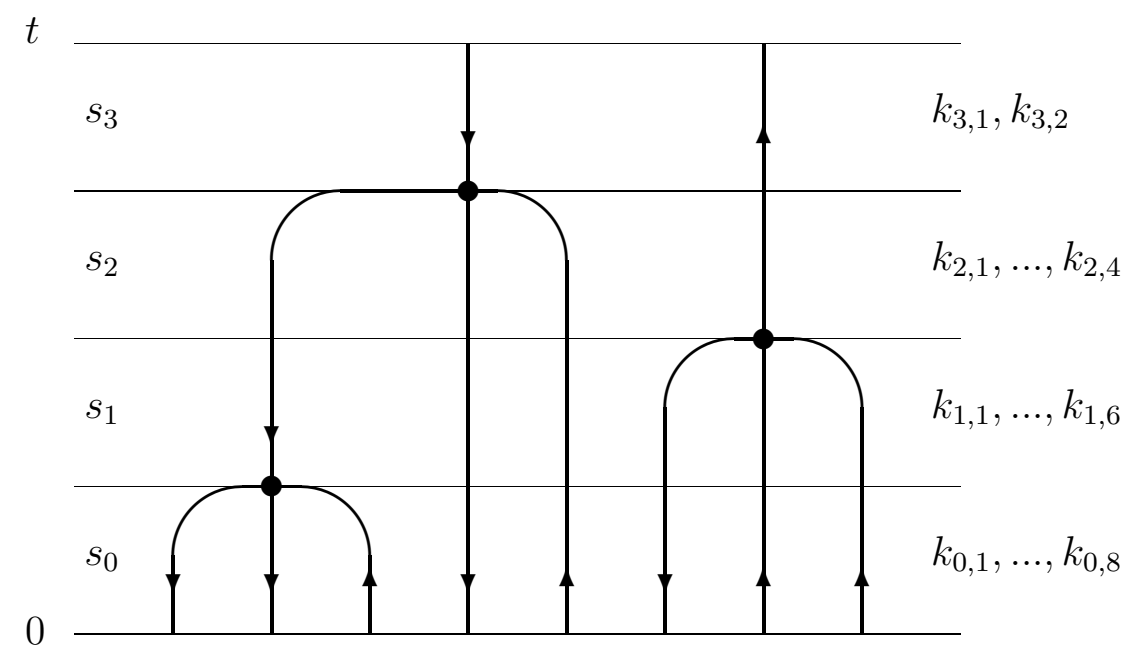

Figure 1: A Feynman diagram with $n_{0}=2, n=3$. Up (down) arrows correspond to parity $+1(-1)$. The operator ordering at $t=0$ is $a^{*} a^{*} a a^{*} a a^{*} a a$. The interaction history is $(1,4,1)$.

which, using (2.20), integrates in time to

$$
\begin{aligned}
& \prod_{j=1}^{n} \hat{a}\left(k_{j}, \sigma_{j}, t\right)=\prod_{j=1}^{n} \hat{a}\left(k_{j}, \sigma_{j}\right)-\mathrm{i} \lambda \sum_{m=1}^{n} \sigma_{m} \int_{0}^{t} d s \int_{\left(\mathbb{T}^{d}\right)^{3}} d k_{2}^{\prime} d k_{3}^{\prime} d k_{4}^{\prime} \\
& \quad \times \delta\left(k_{m}-k_{2}^{\prime}-k_{3}^{\prime}-k_{4}^{\prime}\right) \frac{1}{2}\left(\left(1+\sigma_{m}\right) \widehat{V}\left(k_{2}^{\prime}+k_{3}^{\prime}\right)+\left(1-\sigma_{m}\right) \widehat{V}\left(k_{3}^{\prime}+k_{4}^{\prime}\right)\right) \\
& \quad \times \exp \left[-\mathrm{i} s\left(-\sigma_{m} \omega\left(k_{m}\right)-\omega\left(k_{2}^{\prime}\right)+\sigma_{m} \omega\left(k_{3}^{\prime}\right)+\omega\left(k_{4}^{\prime}\right)\right)\right] \\
& \quad \times\left(\prod_{j=1}^{m-1} \hat{a}\left(k_{j}, \sigma_{j}, s\right)\right) \hat{a}\left(k_{2}^{\prime},-1, s\right) \hat{a}\left(k_{3}^{\prime}, \sigma_{m}, s\right) \hat{a}\left(k_{4}^{\prime}, 1, s\right)\left(\prod_{j=m+1}^{n} \hat{a}\left(k_{j}, \sigma_{j}, s\right)\right) .
\end{aligned}
$$

Here the products are ordered from left to right with increasing label $j$.

Iteration of (2.22) yields the Duhamel expansion for products of the form

$$
\prod_{j=1}^{n_{0}} \hat{a}\left(k_{j}, \sigma_{j}, t\right), \quad t>0 .
$$

The expansion is most concisely organized through Feynman diagrams, compare with Fig. 1. We explain a generic term.

We denote the final time by $t, t>0$. The initial time is 0 . There will be $n$ "collisions" or interactions, $n \geq 0$, which in the Feynman diagram are represented as fusions. We subdivide $[0, t]$ into $n+1$ time slices with index $i \in I_{0, n}=\{0, \ldots, n\}$. Omitting the zero we set $I_{n}=\{1, \ldots, n\}$. The $i$-th slice has length $s_{i}$. Thus $\sum_{i=0}^{n} s_{i}=t$ and the slices are $\left[0, s_{0}\right], \ldots,\left[s_{0}+\ldots+s_{n-1}, t\right]$. To describe the fusions we start in slice 0 with $n_{0}+2 n$ line segments, see Fig. 1 for the orientation of the time axis. At time $t=s_{0}$ exactly one 
triplet of neighboring line segments fuses into a single line segment, while all remaining line segments are continued vertically. Thus in time slice 1 one has exactly $n_{0}+2 n-2$ line segments. At time $t=s_{0}+s_{1}$ exactly one triplet of neighboring line segments fuses into a single line segment, etc.. The last fusion is at $t=s_{0}+\ldots+s_{n-1}$ and in slice $n$ one has $n_{0}$ line segments. Sometimes it is convenient to read the Feynman diagram backwards in time. The only change is that "fusion" is turned into "branching". We now label the line segments by momenta $k_{i, j} \in \mathbb{T}^{d}$ and by parities $\sigma_{i, j} \in\{-1,1\}$. The first index is the label of the slice. In the $i$-th slice we label the momenta and parities from left to right by $j=1, \ldots, m_{i}$ with $m_{i}=n_{0}+2 n-2 i$. The corresponding index set is denoted by $\mathcal{I}_{n ; n_{0}}=\left\{(i, j) \mid 0 \leq i \leq n, 1 \leq j \leq m_{i}\right\}$. An interaction history is denoted by $\ell=\left(\ell_{1}, \ldots, \ell_{n}\right)$, where $\ell_{i}$ refers to the index of the fused line segment in slice $i$, which is also the index of the leftmost line segment in the fusing triplet in slice $i-1$. The set of all interaction histories is denoted by $G_{n}$. Clearly $G_{0}=\emptyset$ and $G_{n}=I_{m_{1}} \times \ldots \times I_{m_{n}}$.

With these preparations the $n$-th term of the expansion reads

$$
\begin{aligned}
\mathcal{F}_{n}( & \left.t, k_{n, 1}, \ldots, k_{n, n_{0}}, \sigma_{n, 1}, \ldots, \sigma_{n, n_{0}}\right)[\hat{a}] \\
= & (-\mathrm{i} \lambda)^{n} \sum_{\ell \in G_{n}} \sum_{\sigma \in\{-1,1\}^{\mathcal{I}_{n ; n_{0}}}} \int_{\left(\mathbb{T}^{d}\right)^{\mathcal{I}_{n ; n_{0}}}} d k \Delta_{n, \ell}(k, \sigma) \\
& \times \prod_{i=1}^{n}\left\{\frac{1}{2}\left(1+\sigma_{i, \ell_{i}}\right) \hat{V}\left(k_{i-1, \ell_{i}}+k_{i-1, \ell_{i}+1}\right)+\frac{1}{2}\left(1-\sigma_{i, \ell_{i}}\right) \hat{V}\left(k_{i-1, \ell_{i}+1}+k_{i-1, \ell_{i}+2}\right)\right\} \\
& \times \prod_{j=1}^{n_{0}+2 n} \hat{a}\left(k_{0, j}, \sigma_{0, j}\right) \int_{\left(\mathbb{R}_{+}\right)^{I_{0, n}}} d \underline{s} \delta\left(t-\sum_{i=0}^{n} s_{i}\right) \prod_{i=1}^{n} \exp \left[-\mathrm{i} t_{i}(\underline{s}) \Omega_{i-1 ; \ell_{i}}\left(k, \sigma_{i, \ell_{i}}\right)\right]
\end{aligned}
$$

as a polynomial of order $n_{0}+2 n$ in the initial fields. Here we have introduced the following shorthands,

$$
t_{i}(\underline{s})=\sum_{j=0}^{i-1} s_{j}, \quad i=1, \ldots, n
$$

and

$$
\Omega_{i ; j}(k, \sigma)=-\sigma \omega\left(k_{i, j}+k_{i, j+1}+k_{i, j+2}\right)-\omega\left(k_{i, j}\right)+\sigma \omega\left(k_{i, j+1}\right)+\omega\left(k_{i, j+2}\right) .
$$

$\Delta_{n, \ell}$ contains the $\delta$-functions restricting the integral over $k$ and the sum over $\sigma$ to the graph defined by the interaction history $\ell$. As can be seen from (2.22), for non-fusing line segments the momentum and the parity are transported unchanged, while the triplet of neighboring line segments with parities,,-++ fuses into a line segment with parity + and the triplet of neighboring line segments with parities,,--+ fuses into a line segment with parity - . In other words, the middle line conserves parity, while the left neighbor carries parity - and the right neighbor parity + . Thus, if the parities in slice $n$ are given, then they are already determined for the remaining diagram. At a fusion the 
total momentum is conserved (Kirchhoff's rule). Explicitly,

$$
\begin{aligned}
\Delta_{n, \ell}(k, \sigma) & =\prod_{i=1}^{n}\left\{\prod_{j=1}^{\ell_{i}-1}\left[\delta\left(k_{i, j}-k_{i-1, j}\right) \mathbb{1}\left(\sigma_{i, j}=\sigma_{i-1, j}\right)\right]\right. \\
& \times \delta\left(k_{i, \ell_{i}}-\sum_{j^{\prime}=0}^{2} k_{i-1, \ell_{i}+j^{\prime}}\right) \mathbb{1}\left(\sigma_{i-1, \ell_{i}}=-1\right) \mathbb{1}\left(\sigma_{i-1, \ell_{i+1}}=\sigma_{i, \ell_{i}}\right) \mathbb{1}\left(\sigma_{i-1, \ell_{i+2}}=1\right) \\
& \left.\times \prod_{j=\ell_{i}+1}^{m_{i}}\left[\delta\left(k_{i, j}-k_{i-1, j+2}\right) \mathbb{1}\left(\sigma_{i, j}=\sigma_{i-1, j+2}\right)\right]\right\}
\end{aligned}
$$

with $\mathbb{1}$ the symbol for the indicator function.

The Duhamel expansion of the product (2.23) cut at order $N$ is given by

$$
\begin{aligned}
\prod_{j=1}^{n_{0}} \hat{a}\left(k_{j}, \sigma_{j}, t\right)= & \sum_{n=0}^{N-1} \mathcal{F}_{n}\left(t, k_{1}, \ldots, k_{n_{0}}, \sigma_{1}, \ldots, \sigma_{n_{0}}\right)[\hat{a}] \\
& +\int_{0}^{t} d s \mathcal{F}_{N}\left(t-s, k_{1}, \ldots, k_{n_{0}}, \sigma_{1}, \ldots, \sigma_{n_{0}}\right)[\hat{a}(s)] .
\end{aligned}
$$

As before, in (2.28) $\hat{a}$ denotes the time 0 field, while $\hat{a}(s)$ is the time $s$ field as it appears in (2.22). To complete the Feynman diagrams the expansion in (2.28) has still to be averaged over some initial state. Note (i) the error term still contains the full time evolution and (ii) the operator order for the product appearing in $\mathcal{F}_{n}$ depends on the particular interaction history.

Given an initial quasifree state of the form (2.17) with bounded $W$, one can estimate roughly the magnitude of a Feynman diagram with respect to this initial state. As an example, let us consider $\left\langle\hat{a}(k, t)^{*} \hat{a}\left(k^{\prime}, t\right)\right\rangle$ for which $n_{0}=2$. At order $n$ of the expansion, from the momentum and time integrations one obtains the bound $\lambda^{n} t^{n} c^{n} / n$ ! with a suitable constant $c$. The initial state has $n$ ! terms of the same size. Thus a Feynman diagram is bounded by $\lambda^{n} t^{n} c^{n}$. However the number of collision histories is also $n$ !, thus yielding a zero radius of convergence for the infinite series.

One possibility to improve the situation would be to extract some extra decay in time. A point in case is a Fermi fluid where particles interact only if they are inside a prescribed bounded region. Outside this region they do not interact which generates a controllable decay. As proved in [22, 23] the Duhamel expansion converges in operator norm provided the coupling strength satisfies $|\lambda|<\lambda_{0}$ with small $\lambda_{0}$.

For the kinetic limit no such improved time decay seems to be available and one is left with the following tentative program.

1.) One studies the convergence of each Feynman diagram for rescaled time $\lambda^{-2} t, t>0$, in the limit $\lambda \rightarrow 0$.

2.) Following the pioneering work of Erdös and Yau [24] in case of the linear Schrödinger equation with a weak random potential, one cuts the series at some $\lambda$-dependent $N$, the generic choice being $\lambda N$ ! = 1 , or perhaps $\lambda^{\kappa} N !=1$ with a suitable choice of $\kappa>0$, and 
tries to control the error term by some other means. In combination with 1.) this would allow one to determine the two-point function

$$
\left\langle\hat{a}\left(k, \lambda^{-2} t\right)^{*} \hat{a}\left(k^{\prime}, \lambda^{-2} t\right)\right\rangle \quad \text { as } \lambda \rightarrow 0 .
$$

We will argue that step 1 can be carried out under specified conditions on the dispersion relation $\omega$. For step 2, at present our only other help is stationarity. This means that we take as initial state $\langle\cdot\rangle$ the KMS state for $H$ with a suitable choice of the chemical potential. Of course, 2.29) is then time-independent. Quantities of interest would be twotime correlations in equilibrium as $\left\langle\hat{a}\left(k, \lambda^{-2} t\right)^{*} \hat{a}\left(k^{\prime}\right)\right\rangle$ and, considerably more difficult to handle, the number density time correlation $\left\langle\hat{a}\left(k, \lambda^{-2} t\right)^{*} \hat{a}\left(k, \lambda^{-2} t\right) \hat{a}\left(k^{\prime}\right)^{*} \hat{a}\left(k^{\prime}\right)\right\rangle$.

\section{The quantum BBGKY hierarchy}

We return to position space. The $n$-th reduced density matrix, at time $t$ is given by the expectation

$$
\rho_{n}\left(x_{1}, y_{1}, \ldots, x_{n}, y_{n}, t\right)=\left\langle\left(\prod_{i=1}^{n} a\left(y_{i}, t\right)^{*}\right)\left(\prod_{j=n}^{1} a\left(x_{j}, t\right)\right)\right\rangle
$$

Here $\rho_{n}(t)$ should be regarded as the kernel of a positive operator acting on $\ell_{2}\left(\mathbb{Z}^{d}\right)^{\otimes n}$ via summation over $y$.

For both fermions and bosons $\rho_{n}(t)$ is symmetric in the arguments $\left(x_{j}, y_{j}\right), j=$ $1, \ldots, n$. In addition, for fermions $\rho_{n}(t)$ is separately antisymmetric in the arguments $\left(x_{1}, \ldots, x_{n}\right)$ and $\left(y_{1}, \ldots, y_{n}\right)$, while it is symmetric for bosons. To differentiate $\rho_{n}(t)$ in time we use (2.12). The resulting operator ordering is no longer normal, in general, and one has to normal order so as to get a closed evolution equation for the $\rho_{n}(t)$ 's. Let us define, as operators on $\ell_{2}\left(\mathbb{Z}^{d}\right)^{\otimes n}$,

$$
H^{(n)}=H_{0}^{(n)}+\lambda V^{(n)}
$$

with

$$
H_{0}^{(n)} \psi_{n}\left(x_{1}, \ldots, x_{n}\right)=\sum_{j=1}^{n} \sum_{w \in \mathbb{Z}^{d}} \alpha\left(x_{j}-w\right) \psi_{n}\left(x_{1}, \ldots, w, \ldots, x_{n}\right)
$$

and

$$
V^{(n)} \psi_{n}\left(x_{1}, \ldots, x_{n}\right)=\frac{1}{2} \sum_{i \neq j=1}^{n} V\left(x_{i}-x_{j}\right) \psi_{n}\left(x_{1}, \ldots, x_{n}\right) .
$$

We also define

$$
\left(C_{n, n+1} \rho_{n+1}\right)\left(x_{1}, \ldots, y_{n}\right)=-\mathrm{i} \sum_{j=1}^{n} \sum_{w \in \mathbb{Z}^{d}}\left(V\left(x_{j}-w\right)-V\left(y_{j}-w\right)\right) \rho_{n+1}\left(x_{1}, \ldots, y_{n}, w, w\right) \text {. }
$$


Then the reduced density matrices satisfy

$$
\frac{d}{d t} \rho_{n}(t)=-\mathrm{i}\left[H^{(n)}, \rho_{n}(t)\right]+\lambda C_{n, n+1} \rho_{n+1}(t)
$$

which is the quantum BBGKY hierarchy.

On purely mathematical grounds, the unitary evolution extends naturally to the full Fock space $\mathfrak{F}$. A state on $\mathfrak{F}$ is given through some positive density matrix $S$ with $\operatorname{tr}_{\mathfrak{F}} S=1$. We require gauge invariance as $\left[S, e^{i \vartheta N}\right]=0$ for all $\vartheta \in[0,2 \pi]$ with $N$ the number operator on $\mathfrak{F}$. Thus, denoting by $P_{n}$ the projection onto the $n$-particle subspace $\mathfrak{F}_{n}$, it holds

$$
P_{m} S P_{n}=\delta_{m n} S_{n}
$$

$S_{n}$ is a positive operator on $\mathfrak{F}_{n}$ and $\operatorname{tr}_{\mathfrak{F}_{n}} S_{n} \leq 1$. Since particles are taken to be indistinguishable, we require $S_{n}$ to be permutation invariant as an operator on $\mathfrak{F}_{n}$. Let us denote by $\operatorname{tr}_{[m, n]}, m \leq n$, the partial trace on $\mathfrak{F}_{n}$ over the tensor product factors with labels $m$ to $n$, in particular $\operatorname{tr}_{[1, n]}=\operatorname{tr}_{\mathfrak{F}_{n}}$. In this general context the $n$-th reduced density matrix is defined through

$$
\rho_{n}=\sum_{m=0}^{\infty} \frac{(n+m) !}{m !} \operatorname{tr}_{[n+1, n+m]} S_{n+m}
$$

as a positive operator on $\mathfrak{F}_{n}$, with the convention $\operatorname{tr}_{[n+1, n]} S_{n}=S_{n}$. The normalization is such that

$$
\operatorname{tr}_{[1, n]} \rho_{n}=\operatorname{tr}_{\mathfrak{F}}[S N(N-1) \ldots(N-n+1)],
$$

which is assumed to be bounded by $c^{n}$. Then (3.8) can be inverted as

$$
S_{n}=\sum_{m=0}^{\infty} \frac{(-1)^{m}}{n ! m !} \operatorname{tr}_{[n+1, n+m]} \rho_{n+m} .
$$

If $\operatorname{tr}_{\mathfrak{F}}\left[P_{\theta} S\right]=1, \theta= \pm 1$, then, using the realization of $a(x), a(x)^{*}$ on Fock space, it is easy to check that (3.8) agrees with the definition (3.1).

The density matrix $S$ evolves in time through

$$
P_{m} S(t) P_{n}=\delta_{m n} \mathrm{e}^{-\mathrm{i} H^{(n)} t} S_{n} \mathrm{e}^{\mathrm{i} H^{(n)} t} .
$$

We insert in the definition (3.8) and differentiate with respect to $t$. The permutation symmetry of the state is preserved in time. This leads to

$$
\frac{d}{d t} \rho_{n}(t)=-\mathrm{i}\left[H^{(n)}, \rho_{n}(t)\right]-\mathrm{i} \lambda \sum_{j=1}^{n} \operatorname{tr}_{[n+1, n+1]}\left(\left[V_{j, n+1}, \rho_{n+1}(t)\right]\right),
$$

where $V_{j, n+1}$ is multiplication by $V\left(x_{j}-x_{n+1}\right)$ as an operator on $\mathfrak{F}_{n+1}$. Clearly, (3.12) is identical to (3.6).

The time-integrated version of (3.6) reads

$$
\rho_{n}(t)=\mathrm{e}^{-\mathrm{i} H^{(n)} t} \rho_{n} \mathrm{e}^{\mathrm{i} H^{(n)} t}+\lambda \int_{0}^{t} d s \mathrm{e}^{-\mathrm{i} H^{(n)}(t-s)} C_{n, n+1} \rho_{n+1}(s) \mathrm{e}^{\mathrm{i} H^{(n)}(t-s)} .
$$


Of interest is $\rho_{1}(t)$. Its perturbation series is generated by iterating (3.13). This will not be an expansion in $\lambda$, since $H^{(n)}$ depends itself on $\lambda$. Expanding $\exp \left[-\mathrm{i} t\left(H_{0}^{(n)}+\lambda V^{(n)}\right)\right]$ with respect to $\lambda$ and inserting in (3.13) yields a perturbation expansion in $\lambda$. For bosons and fermions it has to be in one-to-one correspondence with the Feynman diagrams of Section 2. By construction, for the BBGKY hierarchy the expectation with respect to the initial state is over a normal ordered product of operators, while for Feynman diagrams normal order does not hold, in general, compare with (2.24). On the other hand, reading Feynman diagrams backwards in time, single line segments can branch only into three line segments, while the perturbation expansion of the BBGKY hierarchy in the form (3.13) consists of $n$ particles interacting amongst themselves and one extra particle added through the "collision" $C_{n, n+1}$.

The BBGKY hierarchy is used by Benedetto et al. [25, 26, 27] in their study of the kinetic limit. One observes that for the free evolution generated by $H_{0}^{(n)}$ the kinetic limit, space $\sim \lambda^{-2}$, time $\sim \lambda^{-2}$, is equivalent to the semiclassical limit. This can be exploited by transforming $\rho_{n}$ in each of its variables $\left(x_{j}, y_{j}\right)$ to a Wigner function. Thereby the BBGKY hierarchy turns into a hierarchy of multi-point Wigner functions. The free part corresponds to classical particles with kinetic energy $\omega(k)$ and the difficulty resides in handling the nonlocal "collisions". Benedetto et al. work in the continuum, $\mathbb{R}^{3}$ instead of $\mathbb{Z}^{3}$, and use the quadratic dispersion law $\omega(k)=k^{2}$. The initial reduced density matrices are assumed to be of the factorized form

$$
\rho_{n}\left(x_{1}, y_{1}, \ldots, x_{n}, y_{n}\right)=\prod_{j=1}^{n} \rho_{1}\left(x_{j}, y_{j}\right)
$$

at least asymptotically for small $\lambda$. (3.14) does not have the antisymmetry required for fermions. For bosons the equality $\rho_{2}=\rho_{1} \otimes \rho_{1}$ forces $\rho_{1}$ to be a pure state as can be seen from

Remark 3.1 (Factorization). Using the spectral representation of $\rho_{1}$ with eigenfunctions $\phi_{j}$ and eigenvalues $\lambda_{j} \geq 0$, symmetry in $x_{1}, x_{2}$ implies

$$
\sum_{i, j=1}^{\infty} \lambda_{i} \lambda_{j} \phi_{i}\left(x_{1}\right) \phi_{i}\left(y_{1}\right)^{*} \phi_{j}\left(x_{2}\right) \phi_{j}\left(y_{2}\right)^{*}=\sum_{i, j=1}^{\infty} \lambda_{i} \lambda_{j} \phi_{j}\left(x_{1}\right) \phi_{i}\left(y_{1}\right)^{*} \phi_{i}\left(x_{2}\right) \phi_{j}\left(y_{2}\right)^{*}
$$

Taking the inner product with $\phi_{\alpha}\left(x_{2}\right)$ from left and right yields $\lambda_{\alpha}\left(1-\lambda_{\alpha}\right)=0$.

$\rho_{1}$ defines the scaled Wigner function through

$$
W_{\lambda}(r, v, t)=\int d \eta \mathrm{e}^{\mathrm{i} \eta \cdot v} \rho_{1}\left(\lambda^{-2} r+\frac{1}{2} \eta, \lambda^{-2} r-\frac{1}{2} \eta, t\right) .
$$

It is assumed that, for $t=0$,

$$
\lim _{\lambda \rightarrow 0} W_{\lambda}(r, v)=W_{0}(r, v)
$$


with $W_{0}(r, v)$ sufficiently smooth and of rapid decay in both arguments. Benedetto et al. prove that the perturbation series for $W_{\lambda}\left(r, v, \lambda^{-2} t\right)$ converges to a limit term by term. We describe their limit in Section 5. The convergence imposed in (3.17) cannot hold for a sequence of Wigner functions coming from a pure state. The factorization (3.14) is satisfied only for states which have some support in $\left(1-P_{+}-P_{-}\right) \mathfrak{F}$ and thus rules out bosons and fermions. (3.14) is a property characteristic for boltzmannions.

For bosons and fermions one can switch freely between the BBGKY hierarchy and the Duhamel expansion of Section 2, Once we assume the factorization (3.14), the BBGKY hierarchy refers to a larger class of states, not restricted to $\left(P_{+}+P_{-}\right) \mathfrak{F}$, and the mapping to the Duhamel expansion of Section 2 is lost. Thus in the work of Benedetto et al. some of their oscillatory integrals reappear in the Feynman diagrams of the Duhamel expansion. But there are still other diagrams. Conversely Benedetto et al. have to consider oscillatory integrals which do not correspond to any of the Feynman diagrams studied here.

\section{A comparison with the weakly nonlinear Schrödinger equation}

For the Hamiltonian (2.3) we regard $a(x)$ as a complex-valued commutative field and, to distinguish, denote it by $\psi: \mathbb{Z}^{d} \rightarrow \mathbb{C}$. The classical Hamiltonian functional reads

$$
H=\sum_{x, y \in \mathbb{Z}^{d}} \alpha(x-y) \psi(x)^{*} \psi(y)+\frac{1}{2} \lambda \sum_{x, y \in \mathbb{Z}^{d}} V(x-y)|\psi(x)|^{2}|\psi(y)|^{2} .
$$

We set

$$
\psi(x)=\frac{1}{\sqrt{2}}\left(q_{x}+\mathrm{i} p_{x}\right)
$$

and regard $q_{x}, p_{x}$ as canonically conjugate variables. Then the field $\psi$ evolves in time as

$$
\frac{d}{d t} \psi(x, t)=-\mathrm{i} \sum_{y \in \mathbb{Z}^{d}} \alpha(x-y) \psi(y, t)-\mathrm{i} \lambda \sum_{y \in \mathbb{Z}^{d}} V(x-y)|\psi(y, t)|^{2} \psi(x, t),
$$

which has the same form as (2.12). In particular, using the interaction representation as in (2.19), the Duhamel expansion for products of the form $\prod_{j=1}^{n_{0}} \hat{\psi}\left(k_{j}, \sigma_{j}, t\right)$ is identical to (2.28) derived for the quantum evolution. The only difference resides in the average over the initial state. For the quantum case one has

$$
\left\langle\prod_{j=1}^{n_{0}+2 n} \hat{a}\left(k_{j}, \sigma_{j}\right)\right\rangle
$$

with an operator ordering inherited from the Feynman diagram under consideration, while in the classical case one has to substitute (4.4) by

$$
\left\langle\prod_{j=1}^{n_{0}+2 n} \hat{\psi}\left(k_{j}, \sigma_{j}\right)\right\rangle
$$


where $\langle\cdot\rangle$ denotes the average over a suitable initial probability measure on the $\psi$-field. Since the $\psi$-field is commutative, the ordering is irrelevant.

Let us pursue this difference in more detail. For a quasifree state it holds, compare with Definition 2.1 and Appendix A,

$$
\left\langle\prod_{j=1}^{2 n} \hat{a}\left(k_{j}, \sigma_{j}\right)\right\rangle=\sum_{\pi \in \mathfrak{P}(2 n)} \varepsilon(\pi) \prod_{j=1}^{n}\left\langle\hat{a}\left(k_{\pi(j)}, \sigma_{\pi(j)}\right) \hat{a}\left(k_{\pi(n+j)}, \sigma_{\pi(n+j)}\right)\right\rangle .
$$

Here $\mathfrak{P}(2 n)$ is the set of all pairings of $2 n$ elements with labeling such that, in each factor of the product, the operator order is the same as on the left hand side and $\varepsilon(\pi)=1$ for bosons, $\varepsilon(\pi)= \pm 1$ for fermions depending on whether the permutation induced by the pairing is even or odd. Since $\left\langle a^{*} a^{*}\right\rangle=0=\langle a a\rangle$, the average vanishes whenever $\sum_{j=1}^{2 n} \sigma_{j} \neq 0$. The classical analogue of a quasifree state is a Gaussian measure for which the only nonvanishing moments are of the form

$$
\left\langle\prod_{j=1}^{n} \hat{\psi}\left(k_{j}\right)^{*} \hat{\psi}\left(k_{n+j}\right)\right\rangle=\sum_{\pi \in \mathcal{P}(n)} \prod_{j=1}^{n}\left\langle\hat{\psi}\left(k_{j}\right)^{*} \hat{\psi}\left(k_{n+\pi(j)}\right)\right\rangle
$$

with $\mathcal{P}(n)$ denoting the set of all permutations of $n$ elements. Note that (4.7) agrees with the bosonic version of (4.6) except for operator ordering.

For bosons it holds

$$
\left\langle\hat{a}(k)^{*} \hat{a}\left(k^{\prime}\right)\right\rangle=\delta\left(k-k^{\prime}\right) W(k),\left\langle\hat{a}\left(k^{\prime}\right) \hat{a}(k)^{*}\right\rangle=\delta\left(k-k^{\prime}\right)(1+W(k))
$$

and for fermions

$$
\left\langle\hat{a}(k)^{*} \hat{a}\left(k^{\prime}\right)\right\rangle=\delta\left(k-k^{\prime}\right) W(k),\left\langle\hat{a}\left(k^{\prime}\right) \hat{a}(k)^{*}\right\rangle=\delta\left(k-k^{\prime}\right)(1-W(k)) .
$$

If $W$ is smooth, then so is $1+\theta W(k)$, and for a subleading Feynman diagram the particular operator order makes no difference. On the other hand for a leading diagram one has to keep track of the order and the limit will differ classically and quantum mechanically, as it should be. In fact, the collision operator of the Boltzmann equation is purely cubic for the commutative field while it picks up an additional quadratic piece quantum mechanically with a relative sign which depends on the statistics of the particles.

Remark 4.1 (Rayleigh-Jeans catastrophe). If one replaces $\mathbb{Z}^{d}$ by $\mathbb{R}^{d}$ and $\Omega *$ by $-\Delta$, then (4.3) turns into the Hartree equation

$$
\mathrm{i} \frac{\partial}{\partial t} \psi(x, t)=-\Delta \psi(x, t)+\lambda \int_{\mathbb{R}^{d}} d y V(x-y)|\psi(y, t)|^{2} \psi(x, t),
$$

and for a $\delta$-potential into the dispersive nonlinear Schrödinger equation (also called GrossPitaevskii equation)

$$
\mathrm{i} \frac{\partial}{\partial t} \psi(x, t)=-\Delta \psi(x, t)+\lambda|\psi(x, t)|^{2} \psi(x, t) .
$$


At $\lambda=0$ the corresponding equilibrium measure is Gaussian, gauge-invariant, and has the covariance

$$
\left\langle\hat{\psi}(k)^{*} \hat{\psi}\left(k^{\prime}\right)\right\rangle=\delta\left(k-k^{\prime}\right)\left(\beta\left(k^{2}-\mu\right)\right)^{-1}, \quad \mu<0
$$

For dimension $d=3$, the covariance (4.12) is ultraviolet divergent. This is the analogue of the classical Rayleigh-Jeans catastrophe for the Maxwell field, in which case the covariance is $\delta\left(k-k^{\prime}\right)|k|^{-1}$.

\section{The spatially homogeneous Boltzmann-Nordheim equa- tion}

We consider an initial state which is quasifree, gauge and translation invariant, and thus completely characterized by its two-point function

$$
\left\langle\hat{a}(k)^{*} \hat{a}\left(k^{\prime}\right)\right\rangle=\delta\left(k-k^{\prime}\right) W(k) .
$$

By construction $W \geq 0$ and for fermions $W \leq 1$ in addition. The dynamics preserves gauge and translation invariance. Therefore

$$
\left\langle\hat{a}(k, t)^{*} \hat{a}\left(k^{\prime}, t\right)\right\rangle=\delta\left(k-k^{\prime}\right) W_{\lambda}(k, t) .
$$

As argued above, one expects that, for small $\lambda, W_{\lambda}(k, t)$ will in approximation be governed by a nonlinear transport equation.

Conjecture 5.1 Under suitable assumptions on $\omega$ and on the covariance $W$ in (5.1), it holds

$$
\lim _{\lambda \rightarrow 0} W_{\lambda}\left(k, \lambda^{-2} t\right)=W(k, t),
$$

$W(k, 0)=W(k)$, and with this initial condition $W(t)$ satisfies the Boltzmann-Nordheim equation

$$
\frac{\partial}{\partial t} W(k, t)=\mathcal{C}(W(t))(k)
$$

Here the collision operator is given by

$$
\begin{aligned}
\mathcal{C}(W)\left(k_{1}\right)=\pi & \int_{\left(\mathbb{T}^{d}\right)^{3}} d k_{2} d k_{3} d k_{4} \delta\left(k_{1}+k_{2}-k_{3}-k_{4}\right) \delta\left(\omega_{1}+\omega_{2}-\omega_{3}-\omega_{4}\right) \\
& \times\left|\widehat{V}\left(k_{2}-k_{3}\right)+\theta \widehat{V}\left(k_{2}-k_{4}\right)\right|^{2}\left(\tilde{W}_{1} \tilde{W}_{2} W_{3} W_{4}-W_{1} W_{2} \tilde{W}_{3} \tilde{W}_{4}\right) .
\end{aligned}
$$

Since the expressions tend to become lengthy we use, here and in what follows, the standard shorthand $\omega_{j}=\omega\left(k_{j}\right), W_{j}=W\left(k_{j}\right), j=1,2,3,4$.

Remark 5.2 (Further collision operators). Inserting the definition $\tilde{W}=1+\theta W$, the 
Boltzmann-Nordheim collision operator becomes

$$
\begin{aligned}
\mathcal{C}(W)\left(k_{1}\right)=\pi & \int_{\left(\mathbb{T}^{d}\right)^{3}} d k_{2} d k_{3} d k_{4} \delta\left(k_{1}+k_{2}-k_{3}-k_{4}\right) \delta\left(\omega_{1}+\omega_{2}-\omega_{3}-\omega_{4}\right) \\
& \times\left|\widehat{V}\left(k_{2}-k_{3}\right)+\theta \widehat{V}\left(k_{2}-k_{4}\right)\right|^{2}\left(\theta \left(W_{2} W_{3} W_{4}+W_{1} W_{3} W_{4}\right.\right. \\
& \left.\left.-W_{1} W_{2} W_{4}-W_{1} W_{2} W_{3}\right)+W_{3} W_{4}-W_{1} W_{2}\right) .
\end{aligned}
$$

In case of the nonlinear Schrödinger equation, $\hat{a}(k)$ is replaced by the commutative field $\hat{\psi}(k)$. Then $\langle\cdot\rangle$ is a translation and gauge invariant Gaussian measure and $W(k)$ in (5.1) defines its covariance. In this case the collision operator reads

$$
\begin{aligned}
& \mathcal{C}_{\mathrm{NLS}}(W)\left(k_{1}\right)=\pi \int_{\left(\mathbb{T}^{d}\right)^{3}} d k_{2} d k_{3} d k_{4} \delta\left(k_{1}+k_{2}-k_{3}-k_{4}\right) \delta\left(\omega_{1}+\omega_{2}-\omega_{3}-\omega_{4}\right) \\
& \quad \times\left|\widehat{V}\left(k_{2}-k_{3}\right)+\widehat{V}\left(k_{2}-k_{4}\right)\right|^{2}\left(W_{2} W_{3} W_{4}+W_{1} W_{3} W_{4}-W_{1} W_{2} W_{4}-W_{1} W_{2} W_{3}\right)
\end{aligned}
$$

and thus differs from (5.4) with $\theta=1$ only through the quadratic terms.

If one imposes the initial condition (3.14) corresponding to boltzmannions, then the collision operator becomes

$$
\begin{aligned}
\mathcal{C}_{\mathrm{CL}}(W)\left(k_{1}\right)= & 2 \pi \int_{\left(\mathbb{T}^{d}\right)^{3}} d k_{2} d k_{3} d k_{4} \delta\left(k_{1}+k_{2}-k_{3}-k_{4}\right) \delta\left(\omega_{1}+\omega_{2}-\omega_{3}-\omega_{4}\right) . \\
& \times\left|\widehat{V}\left(k_{2}-k_{3}\right)\right|^{2}\left(W_{3} W_{4}-W_{1} W_{2}\right) .
\end{aligned}
$$

In their set-up Benedetto et al. prove (5.8) in the sense that the perturbation series generated by the BBGKY hierarchy (3.6) converges term by term to the perturbation series generated by (5.4) with collision operator $\mathcal{C}_{\mathrm{CL}}$. Since they do not renormalize the dispersion as in (6.11) below, they have to impose that $\widehat{V}(0)=0$. One recognizes (5.8) as the classical Boltzmann equation with the Born approximation to the differential cross section. Thus on the kinetic level boltzmannions behave like classical point particles.

Note that for bosons, $\theta=1$,

$$
\mathcal{C}=\mathcal{C}_{\mathrm{NLS}}+\mathcal{C}_{\mathrm{CL}}
$$

to say, adding the collision operators for classical waves and classical particles yields the quantum mechanical collision operator. It is surprising that quantizing either the nonlinear Schrödinger equation or classical point particles results in such a small modification on the level of the kinetic equation.

Remark 5.3 (Spatially inhomogeneous Boltzmann equation). If one adds in (5.4), (5.5) the spatial variation, then the Boltzmann-Nordheim equation becomes

$$
\frac{\partial}{\partial t} W(r, k, t)+\frac{1}{2 \pi} \nabla_{k} \omega(k) \cdot \nabla_{r} W(r, k, t)=\mathcal{C}(W(r, \cdot, t))(k)
$$


where our notation is supposed to indicate that $\mathcal{C}$ acts on the argument $k$ at fixed $r, t$. Of course, the same holds for $\mathcal{C}$ replaced by $\mathcal{C}_{\mathrm{NLS}}$ or $\mathcal{C}_{\mathrm{CL}}$. (5.10) can be interpreted as coming from the motion of classical particles with kinetic energy $\omega(k)$. Collisions between particles are implicitly defined through the conservation of energy and momentum,

$$
\omega_{1}+\omega_{2}=\omega_{3}+\omega_{4}, \quad k_{1}+k_{2}=k_{3}+k_{4} .
$$

The collision rule thus depends on the particular form of $\omega$ and can be very counterintuitive when viewed from the perspective of potential scattering of mechanical particles. In the case of the special dispersion relation $\omega(k)=k^{2} / 2$ on $\mathbb{R}^{3}$, energy and momentum conservation can be parameterized in the form

$$
k_{3}=k_{1}-\hat{\omega} \cdot\left(k_{1}-k_{2}\right) \hat{\omega}, \quad k_{4}=k_{2}+\hat{\omega} \cdot\left(k_{1}-k_{2}\right) \hat{\omega},
$$

with $|\hat{\omega}|=1$, i.e., $\hat{\omega} \in S^{2}$. Then the collision operator acquires the more conventional form

$$
\begin{aligned}
\mathcal{C}(W)\left(k_{1}\right) & =\int_{\mathbb{R}^{3}} d k_{2} \int_{S^{2}} d \hat{\omega}\left|\hat{\omega} \cdot\left(k_{1}-k_{2}\right)\right| \mid \widehat{V}\left(\hat{\omega} \cdot\left(k_{1}-k_{2}\right) \hat{\omega}\right) \\
& +\left.\theta \widehat{V}\left(k_{1}-k_{2}-\hat{\omega} \cdot\left(k_{1}-k_{2}\right) \hat{\omega}\right)\right|^{2}\left(\tilde{W}_{1} \tilde{W}_{2} W_{3} W_{4}-W_{1} W_{2} \tilde{W}_{3} \tilde{W}_{4}\right) .
\end{aligned}
$$

For a rotational symmetric potential, $\widehat{V}(k)=\widehat{V}_{\mathrm{r}}(|k|)$, the collision cross section simplifies to

$$
\left|\hat{\omega} \cdot\left(k_{1}-k_{2}\right)\right|\left|\widehat{V}_{\mathrm{r}}\left(\left|\hat{\omega} \cdot\left(k_{1}-k_{2}\right)\right|\right)+\theta \widehat{V}_{r}\left(\left(\left(k_{1}-k_{2}\right)^{2}-\left(\hat{\omega} \cdot\left(k_{1}-k_{2}\right)\right)^{2}\right)^{1 / 2}\right)\right|^{2} .
$$

One notes that for a smooth potential the decay is exponential and even for hard spheres the decay is proportional to $\left|k_{1}-k_{2}\right|^{-3}$. Thus at high energies there is only little scattering.

Remark 5.4 (History). Equations (5.4), (5.5) were first written down by Nordheim [1], where he had in mind the true quantum mechanical scattering cross section, rather than only its Born approximation. In 1929 Peierls [2] studied lattice vibrations with small nonlinearity both classically and quantized. For this particular weakly nonlinear wave equation he derives (5.4) with the analogue of the collision term (5.7). Later on it was realized that Peierls' ideas apply to a more general class of weakly nonlinear wave equations, e.g. see [28]. For quantized lattice vibrations Peierls uses Fermi's golden rule and arrives at (5.4) with the analogue of the collision operator (5.5) for $\theta=1$. The terminology is not uniform. In kinetic theory the name Uehling-Uhlenbeck seems to be most frequent because they studied the equation in their pioneering work [3]. For phonon transport Peierls or Boltzmann-Peierls is used. For dilute Bose gases Boltzmann-Nordheim seems to be rather established. We follow this latter convention for reasons of priority.

To approach the Conjecture one expands $\left\langle\hat{a}(k, t)^{*} \hat{a}\left(k^{\prime}, t\right)\right\rangle$ in the Duhamel series (2.28) up to some $N$ depending suitably on $\lambda$. Currently there seems to be no good idea of how 
to control the error term. This leaves one with the program of the term by term convergence, which to some extent will be explained in Section 6 An important improvement as regards to Section 2 is to renormalize the bare dispersion $\omega$ to

$$
\omega^{\lambda}\left(k_{1}, t\right)=\omega\left(k_{1}\right)+\lambda \int_{\mathbb{T}^{d}} d k_{2} W_{\lambda}\left(k_{2}, t\right)\left(\widehat{V}(0)+\theta \widehat{V}\left(k_{1}-k_{2}\right)\right) .
$$

In contrast to Section 6, in the present context $\omega^{\lambda}$ is time-dependent through the itself unknown $W_{\lambda}(t)$.

By mass conservation

$$
\int_{\mathbb{T}^{d}} d k_{1} W\left(k_{1}, t\right)=\int_{\mathbb{T}^{d}} d k_{1} W\left(k_{1}, 0\right)
$$

Thus the term proportional to $\widehat{V}(0)$ is in fact constant. For the second term one has the trivial estimate

$$
\left|\lambda \int_{\mathbb{T}^{d}} d k_{2} W_{\lambda}\left(k_{2}, t\right) \theta \widehat{V}\left(k_{1}-k_{2}\right)\right| \leq \lambda \int_{\mathbb{T}^{d}} d k_{1} W\left(k_{1}, 0\right) \sum_{x \in \mathbb{Z}^{d}}|V(x)|
$$

These observations leave us with two choices: If $\widehat{V}(k)=\widehat{V}(0)$, then the fermions become noninteracting and one is left with lattice bosons interacting through a quartic on-site potential. $\omega$ is renormalized by a constant proportional to $\lambda$ which is easily taken care off. If $\widehat{V}(k)$ depends on $k$, the situation is more complicated. Because of the convolution, $\omega^{\lambda}$ depends smoothly on $k_{1}$, but it could be rapidly oscillating in $t$. Whether the bound (5.17) suffices to ensure the $\ell_{3}$ dispersivity, the constructive interference, and the crossing bound of Section 6 remains to be investigated.

In this section we discuss a more modest step, namely the leading part of the main term. For this purpose we make the following definitions.

Definition 5.5 (pairing property). Let us consider a Feynman diagram of even order, $n$ even, where the integration over all momentum $\delta$-functions has been carried out. The Feynman diagram satisfies the pairing rule if for every even time slice the momenta are paired, i.e., to each line segment with momentum $k$ and parity $\sigma$ there exists, in the same even time slice another line segment with momentum $-k$ and parity $-\sigma$.

Definition 5.6 (leading diagrams). A Feynman diagram is leading, if it satisfies the pairing property and if it does not contain the factor $\widehat{V}(0)$.

Note that in the 0 -th time slice $\left[0, s_{0}\right]$ the pairing is induced by the initial state. Thus the structure of a leading diagram can be obtained by iteration.

So let us assume that for the time slice with even label $i$ all line segments are paired. If there are $2 n$ line segments, they carry momenta $\sigma_{j} k_{j}, j=1, \ldots, n, \sigma_{j}= \pm 1 . k_{j}$ and $-k_{j}$ are paired. At the end of time slice $i$ three neighboring lines fuse to the line segment with momentum $\sigma^{\prime} k^{\prime}$. If two momenta would be paired, the diagram necessarily contains the factor $\widehat{V}(0)$. Thus we label neighboring lines by three distinct momenta $\sigma_{j} k_{j}$, 


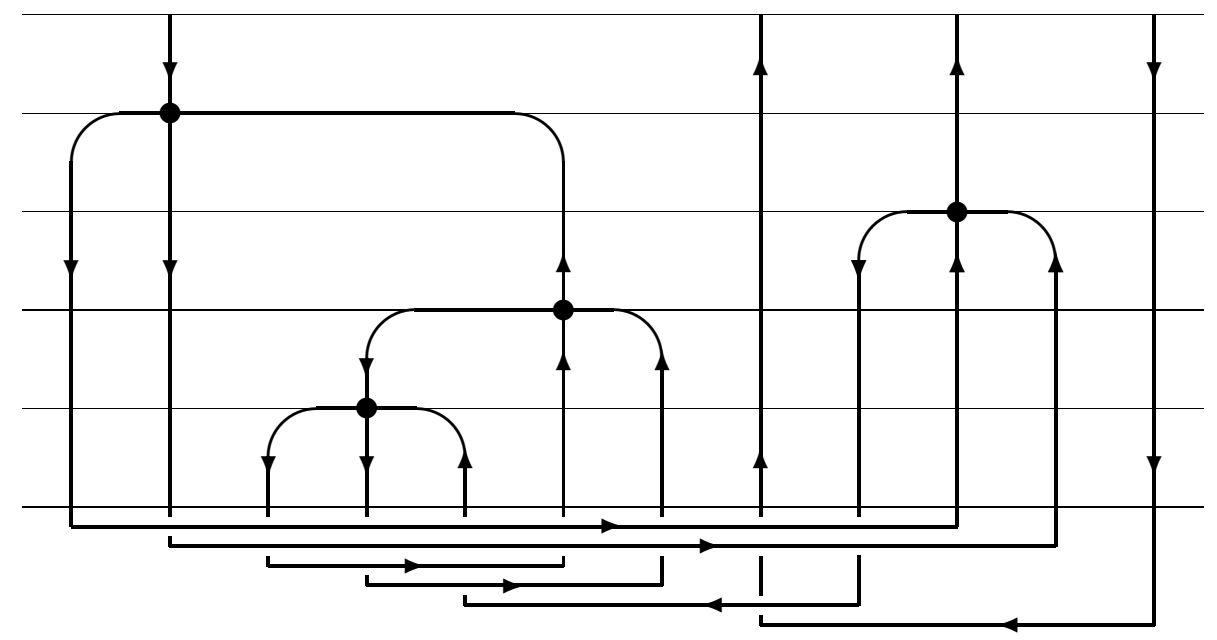

Figure 2: A leading Feynman diagram with $n_{0}=4, n=4$, and interaction history $(3,3,5,1)$. Up (down) arrows correspond to parity $+1(-1)$. The pairing from the initial state is indicated, where right (left) pointing arrow stands for the order $\left\langle a^{*} a\right\rangle\left(\left\langle a a^{*}\right\rangle\right)$.

$j=1,2,3$. In the next step there can be two cases which are both displayed in Fig. 2. Case a) corresponds to the $3^{\text {rd }}$ and $4^{\text {th }}$ fusion, while case b) corresponds to the $1^{\text {st }}$ and $2^{\text {nd }}$ fusion. In case a) the line segment $\sigma^{\prime} k^{\prime}$ does not participate in the fusion at the end of time slice $i+1$. The fusing triplet has momenta $\sigma_{\pi(j)} k_{\pi(j)}, j=1,2,3$, and the momentum of the fused line segment is $\sigma^{\prime \prime} k^{\prime \prime}$. By the same argument as above, the $\pi(j)$ 's must be distinct. To have pairing in time slice $i+2$ requires $k^{\prime}=k^{\prime \prime}, \sigma^{\prime}=-\sigma^{\prime \prime}$. In turn this is possible only if $\{1,2,3\}=\{\pi(1), \pi(2), \pi(3)\}$. In case b) the line segment $\sigma^{\prime} k^{\prime}$ participates in the fusion at the end of time slice $i+1$. The fusing momenta are $\sigma^{\prime} k^{\prime}, \sigma_{\pi(1)} k_{\pi(1)}, \sigma_{\pi(2)} k_{\pi(2)}$. The fused momentum is $\sigma^{\prime \prime} k^{\prime \prime}$. In slice $i+2, \sigma^{\prime \prime} k^{\prime \prime}$ must be paired with, say, $\sigma_{\pi(3)} k_{\pi(3)}$. There will be a factor $\widehat{V}(0)$ unless all three $\pi(j)$ 's are distinct. The pairing of $\sigma^{\prime \prime} k^{\prime \prime}$ and $\sigma_{\pi(3)} k_{\pi(3)}$ is possible only if $\{1,2,3\}=\{\pi(1), \pi(2), \pi(3)\}$.

We conclude that for a leading Feynman diagram there must be three paired line segments in slice $i$ which connect through two fusions to a single paired line segment in slice $i+2, i=0,2, \ldots, n, n$ even, compare with Fig. 2. This property allows us to represent a leading diagram through a contracted diagram which we explain next, see Fig. 3 ,

In a contracted diagram we draw only the even time slices and each pair as a single line segment. A line segment thus carries a momentum $k$. But we still have to distinguish the relative order within the pair. If in the original Feynman diagram the order from left to right is,-+ , then the line carries the order parity $\tau=+1$, while for the order,+- the order parity is $\tau=-1$. In the contracted diagram, at the end of each time slice a triplet of neighboring line segments fuses into a single line segment. In our case, if the leading Feynman diagram has order $2 n$, then each contracted diagram has $n$ collisions and there are $(2 n+1) ! / 2^{n} n$ ! contracted diagrams.

To compute the vertex strength for the contracted diagram one has to sum over all 


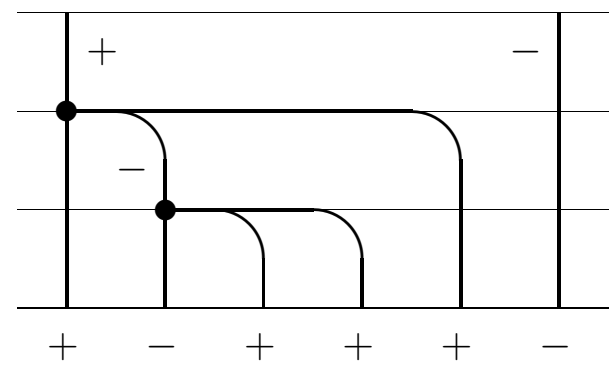

Figure 3: Contraction of the diagram from Figure 2. The order parity, $\tau$, of each contracted line is indicated.

Feynman diagrams which fuse 3 pairs at even time slice into the single pair at time slice $i+2$. There are 16 such diagrams. Their sum yields the vertex strength given below in (5.18).

To write down the integral corresponding to a contracted diagram, it is useful to introduce the "correlation" functions $\widehat{\rho}_{n}:\left(\mathbb{T}^{d} \times\{-1,1\}\right)^{n} \rightarrow \mathbb{C}$ and to define time-dependent collision operators $\mathcal{C}_{j, n+2}(t)$ with $j=1, \ldots, n$. We set

$$
\begin{aligned}
& \left(\mathcal{C}_{j, n+2}(t) \widehat{\rho}_{n+2}\right)\left(k_{1}, \tau_{1}, \ldots, k_{n}, \tau_{n}\right)=2 \int_{\left(\mathbb{T}^{d}\right)^{3}} d g_{2} d g_{3} d g_{4} \delta\left(k_{j}+g_{2}-g_{3}-g_{4}\right) \\
& \quad \times \cos \left(\left(\omega\left(k_{j}\right)+\omega\left(g_{2}\right)-\omega\left(g_{3}\right)-\omega\left(g_{4}\right)\right) t\right) \widehat{V}\left(g_{2}-g_{3}\right)\left(\widehat{V}\left(g_{2}-g_{3}\right)+\theta \widehat{V}\left(g_{2}-g_{4}\right)\right) \\
& \quad \times\left(\widehat{\rho}_{n+2}\left(\ldots, g_{2},-\tau_{j}, \ldots, g_{3}, \tau_{j}, g_{4}, \tau_{j}\right)+\theta \widehat{\rho}_{n+2}\left(\ldots, k_{j}, \tau_{j}, \ldots, g_{3}, 1, g_{4}, 1\right)\right. \\
& \left.\quad-\widehat{\rho}_{n+2}\left(\ldots, k_{j}, \tau_{j}, \ldots, g_{2}, 1, g_{3},-1\right)-\theta \widehat{\rho}_{n+2}\left(\ldots, k_{j}, \tau_{j}, \ldots, g_{2}, 1, g_{4}, 1\right)\right),
\end{aligned}
$$

which acts on the arguments $j, n+1, n+2$ of $\widehat{\rho}_{n+2}$, and

$$
\mathcal{C}_{n+2}(t)=\sum_{j=1}^{n} \mathcal{C}_{j, n+2}(t) \text {. }
$$

Let

$$
\mathcal{M}_{n}^{\lambda}\left(k_{1}, t\right) \delta\left(k_{0}-k_{1}\right)
$$

be the sum over all contracted diagrams at order $n$ with time span $t$. Then

$$
\begin{aligned}
& \mathcal{M}_{n}^{\lambda}\left(k_{1}, \lambda^{-2} t\right) \\
& =\lambda^{2 n} \int_{\left(\mathbb{R}_{+}\right)^{n+1}} d \underline{t} \int_{\left(\mathbb{R}_{+}\right)^{n}} d \underline{s} \delta\left(\sum_{j=1}^{n+1} t_{j}+\sum_{j=1}^{n} s_{j}-\lambda^{-2} t\right)\left(\mathcal{C}_{3}\left(s_{1}\right) \ldots \mathcal{C}_{2 n+1}\left(s_{n}\right) \widehat{\rho}_{2 n+1}\right)\left(k_{1}, 1\right) \\
& =\int_{0 \leq \sum_{j=1}^{n} t_{j} \leq t} d \underline{t} \int_{0 \leq \sum_{j=1}^{n} s_{j} \leq \lambda^{-2}\left(t-\sum_{j=1}^{n} t_{j}\right)} d \underline{s}\left(\mathcal{C}_{3}\left(s_{1}\right) \ldots \mathcal{C}_{2 n+1}\left(s_{n}\right) \widehat{\rho}_{2 n+1}\right)\left(k_{1}, 1\right) .(5.21)
\end{aligned}
$$

In our particular case the input function is given by

$$
\widehat{\rho}_{n}\left(k_{1}, \tau_{1}, \ldots, k_{n}, \tau_{n}\right)=\prod_{j=1}^{n} W\left(k_{j}, \tau_{j}\right)
$$


with

$$
W(k, 1)=W(k), \quad W(k,-1)=1+\theta W(k)=\tilde{W}(k) .
$$

To prove the limit $\lambda \rightarrow 0$ one needs a first assumption on $\omega$.

Assumption A1 $\left(\ell_{3}\right.$ dispersivity). Let us define

$$
p_{t}(x)=\int_{\mathbb{T}^{d}} d k \mathrm{e}^{-\mathrm{i} t \omega(k)} \mathrm{e}^{\mathrm{i} 2 \pi x \cdot k} .
$$

Then there exist $c>0, \delta>0$ such that

$$
\sum_{x \in \mathbb{Z}^{d}}\left|p_{t}(x)\right|^{3} \leq c\langle t\rangle^{-1-\delta}
$$

with the shorthand $\langle t\rangle=\sqrt{1+t^{2}}$.

As in [15] one proves the following bound.

Proposition 5.7 Let $\left\|\rho_{n}\right\|_{1} \leq\left(c_{0}\right)^{n}$, where $\|\cdot\|_{1}$ is the $\ell_{1}\left(\mathbb{Z}^{d}\right)^{\otimes n}$ norm in position space. Then there exists a constant $c$ such that

$$
\int_{0}^{\infty} d \underline{s} \sup _{k_{1} \in \mathbb{T}^{d}}\left|\left(\mathcal{C}_{3}\left(s_{1}\right) \ldots \mathcal{C}_{2 n+1}\left(s_{n}\right) \widehat{\rho}_{2 n+1}\right)\left(k_{1}, 1\right)\right| \leq c^{n} n ! .
$$

Note that $\mathcal{C}_{2 n+1}(s)$ has $2 n-1$ terms of equal size. The $n$ ! in (5.26) thus results from the product of collision operators.

With this bound one can introduce the collision operator

$$
\begin{aligned}
\left(\mathcal{C} \widehat{\rho}_{3}\left(k_{1}, \tau\right)\right. & =2 \pi \int_{\left(\mathbb{T}^{d}\right)^{2}} d k_{2} d k_{3} \delta\left(\omega\left(k_{1}\right)+\omega\left(k_{2}\right)-\omega\left(k_{3}\right)-\omega\left(k_{1}+k_{2}-k_{3}\right)\right) \\
& \times \widehat{V}\left(k_{2}-k_{3}\right)\left(\widehat{V}\left(k_{2}-k_{3}\right)+\theta \widehat{V}\left(k_{2}-k_{4}\right)\right)\left(\widehat{\rho}_{3}\left(k_{2},-\tau, k_{3}, \tau, k_{1}+k_{2}-k_{3}, \tau\right)\right. \\
& +\theta \widehat{\rho}_{3}\left(k_{1}, \tau, k_{2}, 1, k_{1}+k_{2}-k_{3}, 1\right)-\widehat{\rho}_{3}\left(k_{1}, \tau, k_{2}, 1, k_{3},-1\right) \\
& \left.\quad-\theta \widehat{\rho}_{3}\left(k_{1}, \tau, k_{2}, 1, k_{1}+k_{2}-k_{3}, 1\right)\right)
\end{aligned}
$$

where the $\delta$-function is defined through the limit

$$
\delta(\Omega)=\lim _{\varepsilon \rightarrow 0} \frac{1}{\pi} \frac{\varepsilon}{\Omega^{2}+\varepsilon^{2}} .
$$

As before, from $\mathcal{C}$ we construct $\mathcal{C}_{j, n+2}$, which is $\mathcal{C}$ now acting on the variables $k_{j}, k_{n+1}$, $k_{n+2}$, and

$$
\mathcal{C}_{n+2}=\sum_{j=1}^{n} \mathcal{C}_{j, n+2}
$$

Under the conditions of Proposition 5.7 one concludes that

$$
\lim _{\lambda \rightarrow 0} \mathcal{M}_{n}^{\lambda}\left(k_{1}, \lambda^{-2} t\right)=\frac{1}{n !} t^{n}\left(\mathcal{C}_{3} \ldots \mathcal{C}_{2 n+1} \widehat{\rho}_{2 n+1}\right)\left(k_{1}, 1\right)
$$


uniformly in $k_{1}$ and

$$
\sup _{k_{1} \in \mathbb{T}^{d}}\left|\left(\mathcal{C}_{3} \ldots \mathcal{C}_{2 n+1} \widehat{\rho}_{2 n+1}\right)\left(k_{1}, 1\right)\right| \leq c^{n} n !
$$

Thus the sum over $n$ reads

$$
W(k, t)=\sum_{n=0}^{\infty} \frac{t^{n}}{n !}\left(\mathcal{C}_{3} \ldots \mathcal{C}_{2 n+1} \widehat{\rho}_{2 n+1}\right)(k, 1) .
$$

By (5.26) there exists then a $t_{0}$, such that the sum converges provided

$$
0 \leq t<t_{0}
$$

If of interest, the time $t_{0}$ can be computed explicitly. Up to numerical factors of order 1 , $t_{0}$ is proportional to

$$
\sum_{x \in \mathbb{Z}^{d}}|V(x)| \text { and } \int_{0}^{\infty} d t \sum_{x \in \mathbb{Z}^{d}}\left|p_{t}(x)\right|^{3} .
$$

Because of the particular initial conditions (5.22), (5.23), the limit in (5.32) can be written more concisely. One recognizes $W(k, t)$ as the power series solution of the nonlinear Boltzmann-Nordheim equation (5.4) with collision operator

$$
\begin{aligned}
\mathcal{C}(W)\left(k_{1}\right)=2 & \pi \int_{\left(\mathbb{T}^{d}\right)^{3}} d k_{2} d k_{3} d k_{4} \delta\left(k_{1}+k_{2}-k_{3}-k_{4}\right) \delta\left(\omega_{1}+\omega_{2}-\omega_{3}-\omega_{4}\right) \\
& \times \widehat{V}\left(k_{2}-k_{3}\right)\left(\widehat{V}\left(k_{2}-k_{3}\right)+\theta \widehat{V}\left(k_{2}-k_{4}\right)\right) \\
& \times\left(\tilde{W}_{2} W_{3} W_{4}+\theta W_{1} W_{3} W_{4}-W_{1} W_{2} \tilde{W}_{3}-\theta W_{1} W_{2} W_{4}\right)
\end{aligned}
$$

which is identical to (5.5).

\section{Two-point time correlations in thermal equilibrium}

6.1 Set-up. In this and the following section we consider a quantum fluid in thermal equilibrium. We fix some inverse temperature $\beta>0$ and a chemical potential $\mu \in \mathbb{R}$ such that

$$
\omega(k)-\mu>0
$$

for all $k \in \mathbb{T}^{d} \cdot\langle\cdot\rangle_{\beta, \lambda}$ denotes the expectation value with respect to the $\beta$-KMS state for the hamiltonian $H-\mu N, N$ the number operator. The two-point function is denoted by

$$
\begin{aligned}
& \left\langle\hat{a}(k)^{*} \hat{a}\left(k^{\prime}\right)\right\rangle_{\beta, \lambda}=\delta\left(k-k^{\prime}\right) W_{\beta, \lambda}^{\theta}(k), \\
& \left\langle\hat{a}(k) \hat{a}\left(k^{\prime}\right)^{*}\right\rangle_{\beta, \lambda}=\delta\left(k-k^{\prime}\right) \tilde{W}_{\beta, \lambda}^{\theta}(k)=\delta\left(k-k^{\prime}\right)\left(1+\theta W_{\beta, \lambda}^{\theta}(k)\right) .
\end{aligned}
$$

In the limit $\lambda \rightarrow 0$, the state $\langle\cdot\rangle_{\beta, 0}$ is quasifree with

$$
\left\langle\hat{a}(k)^{*} \hat{a}\left(k^{\prime}\right)\right\rangle_{\beta, 0}=\delta\left(k-k^{\prime}\right) W_{\beta}^{\theta}(k) .
$$


Since $W_{\beta}^{\theta}$ is smooth, the two-point function decays exponentially and by quasifreeness all fully truncated correlation functions of order greater than two vanish.

In this section we study the effective propagator as the most basic two-point function, namely

$$
\left\langle\hat{a}(k)^{*} \hat{a}\left(k^{\prime}, t\right)\right\rangle_{\beta, \lambda}=\left\langle\hat{a}(k)^{*} \hat{a}\left(k^{\prime}\right)\right\rangle_{\beta, \lambda} C_{\lambda}(k, t),
$$

which defines $C_{\lambda}(k, t)$ since both sides of (6.4) are proportional to $\delta\left(k-k^{\prime}\right)$. More complicated equilibrium time correlation functions will be discussed in Section 7. Because of the $a$-term, clearly, $C_{\lambda}$ will contain the oscillatory factor $\mathrm{e}^{-\mathrm{i} \omega(k) t}$ varying on time scale 1 . To first order in the Duhamel expansion one has

$$
\begin{aligned}
& \mathrm{e}^{\mathrm{i} \omega\left(k_{1}\right) t}\left\langle\hat{a}\left(k_{0}\right)^{*} \hat{a}\left(k_{1}, t\right)\right\rangle_{\beta, \lambda}=\left\langle\hat{a}\left(k_{0}\right)^{*} \hat{a}\left(k_{1}\right)\right\rangle_{\beta, \lambda} \\
& -\mathrm{i} \lambda \int_{0}^{t} d s \int_{\left(\mathbb{T}^{d}\right)^{3}} d k_{2} d k_{3} d k_{4} \delta\left(k_{1}+k_{2}-k_{3}-k_{4}\right) \widehat{V}\left(k_{2}-k_{3}\right) \\
& \quad \times \exp \left[\mathrm{i} s\left(\omega_{1}+\omega_{2}-\omega_{3}-\omega_{4}\right)\right]\left\langle\hat{a}\left(k_{0}\right)^{*} \hat{a}\left(k_{2}\right)^{*} \hat{a}\left(k_{3}\right) \hat{a}\left(k_{4}\right)\right\rangle_{\beta, \lambda}+\mathcal{O}\left(\lambda^{2}\right) .
\end{aligned}
$$

We write

$$
\begin{aligned}
& \left\langle\hat{a}\left(k_{0}\right)^{*} \hat{a}\left(k_{2}\right)^{*} \hat{a}\left(k_{3}\right) \hat{a}\left(k_{4}\right)\right\rangle_{\beta, \lambda}=\left\langle\hat{a}\left(k_{0}\right)^{*} \hat{a}\left(k_{4}\right)\right\rangle_{\beta, \lambda}\left\langle\hat{a}\left(k_{2}\right)^{*} \hat{a}\left(k_{3}\right)\right\rangle_{\beta, \lambda} \\
& +\theta\left\langle\hat{a}\left(k_{0}\right)^{*} \hat{a}\left(k_{3}\right)\right\rangle_{\beta, \lambda}\left\langle\hat{a}\left(k_{2}\right)^{*} \hat{a}\left(k_{4}\right)\right\rangle_{\beta, \lambda}+\left\langle\hat{a}\left(k_{0}\right)^{*} \hat{a}\left(k_{2}\right)^{*} \hat{a}\left(k_{3}\right) \hat{a}\left(k_{4}\right)\right\rangle_{\beta, \lambda}^{T} .
\end{aligned}
$$

The truncated part is $\mathcal{O}(\lambda)$ and, when first integrated over momenta as in (6.5), is absolutely integrable in $s$. Therefore this contribution will be part of the error term. The first two terms inserted in (6.5) yield $-\mathrm{i} \lambda t\left\langle\hat{a}\left(k_{0}\right)^{*} \hat{a}\left(k_{1}\right)\right\rangle_{\beta, \lambda} R_{\lambda}\left(k_{1}\right)$, where

$$
R_{\lambda}\left(k_{1}\right)=\int_{\mathbb{T}^{d}} d k_{2} W_{\lambda}^{\theta}\left(k_{2}\right)\left(\widehat{V}(0)+\theta \widehat{V}\left(k_{1}-k_{2}\right)\right) .
$$

Our computation suggests that $C_{\lambda}$ has a second oscillatory factor of the form

$$
\mathrm{e}^{-\mathrm{i} \lambda R_{\lambda}(k) t}
$$

varying on the time scale $\lambda^{-1}$. The kinetic time scale is order $\lambda^{-2}$. Thus it suffices to expand up to $\lambda$ as

$$
R_{\lambda}\left(k_{1}\right)=R_{0}\left(k_{1}\right)+\lambda R_{1}\left(k_{1}\right)+\mathcal{O}\left(\lambda^{2}\right)
$$

with

$$
\begin{aligned}
R_{0}\left(k_{1}\right)= & \int_{\mathbb{T}^{d}} d k_{2} W_{\beta}^{\theta}\left(k_{2}\right)\left(\widehat{V}(0)+\theta \widehat{V}\left(k_{1}-k_{2}\right)\right) \\
R_{1}\left(k_{1}\right)= & -\beta \int_{\mathbb{T}^{d}} d k_{2} W_{\beta}^{\theta}\left(k_{2}\right) \tilde{W}_{\beta}^{\theta}\left(k_{2}\right)\left(\widehat{V}(0)+\theta \widehat{V}\left(k_{1}-k_{2}\right)\right) \\
& \times \int_{\mathbb{T}^{d}} d k_{3} W_{\beta}^{\theta}\left(k_{3}\right)\left(\widehat{V}(0)+\theta \widehat{V}\left(k_{1}-k_{3}\right)\right)
\end{aligned}
$$


Potentially the oscillatory term (6.8) could be dangerous because in an expansion in $\lambda$ it may mask the kinetic terms. As we will see, fortunately, $R_{\lambda}$ can be absorbed by renormalizing $\omega$ to

$$
\omega^{\lambda}=\omega+\lambda R_{\lambda} .
$$

The second order term of the Duhamel expansion will not be written out explicitly. It consists of 18 diagrams, 12 of which combine to

$$
-\frac{1}{2} \lambda^{2} t^{2} R_{0}(k)^{2}
$$

The remaining 6 diagrams sum up to

$$
-\delta\left(k-k^{\prime}\right) W_{\beta}^{\theta}(k) \nu(k) \lambda^{2} t
$$

valid for large $t$. The decay coefficient $\nu$ is obtained as

$$
\begin{gathered}
\nu\left(k_{1}\right)=-\int_{0}^{\infty} d t \int_{\left(\mathbb{T}^{d}\right)^{3}} d k_{2} d k_{3} d k_{4} \delta\left(k_{1}+k_{2}-k_{3}-k_{4}\right) \exp \left[\mathrm{i} t\left(\omega_{1}+\omega_{2}-\omega_{3}-\omega_{4}\right)\right] \\
\times \widehat{V}\left(k_{2}-k_{3}\right)\left(\widehat{V}\left(k_{2}-k_{4}\right)+\theta \widehat{V}\left(k_{2}-k_{3}\right)\right)\left(W_{\beta, 3}^{\theta} W_{\beta, 4}^{\theta}-W_{\beta, 2}^{\theta} W_{\beta, 4}^{\theta}-\theta W_{\beta, 2}^{\theta} \tilde{W}_{\beta, 3}^{\theta}\right)
\end{gathered}
$$

with the shorthand $W_{\beta, j}^{\theta}=W_{\beta}^{\theta}\left(k_{j}\right)$. For the real part of $\nu$ one obtains

$$
\begin{aligned}
\Re \nu\left(k_{1}\right)= & \pi \int_{\left(\mathbb{T}^{d}\right)^{3}} d k_{2} d k_{3} d k_{4} \delta\left(k_{1}+k_{2}-k_{3}-k_{4}\right) \delta\left(\omega_{1}+\omega_{2}-\omega_{3}-\omega_{4}\right) \\
& \times \frac{1}{2}\left(\widehat{V}\left(k_{2}-k_{3}\right)+\theta \widehat{V}\left(k_{2}-k_{4}\right)\right)^{2}\left(W_{\beta, 1}^{\theta}\right)^{-1} \tilde{W}_{\beta, 2}^{\theta} W_{\beta, 3}^{\theta} W_{\beta, 4}^{\theta} .
\end{aligned}
$$

In particular

$$
\Re \nu\left(k_{1}\right)>0 .
$$

On the basis of this second order expansion the obvious conjecture is that

$$
C_{\lambda}(k, t) \cong \exp \left[-\left(\mathrm{i} \omega^{\lambda}+\lambda^{2} \nu(k)\right) t\right]
$$

for $t \geq 0$ and $t=\mathcal{O}\left(\lambda^{-2}\right)$, the case $t \leq 0$ following from time reversal as

$$
C_{\lambda}(k, t)^{*}=C_{\lambda}(k,-t) .
$$

Our goal is to prove (6.17). For this purpose we have to assume cluster properties of the equilibrium state and the decay of certain oscillatory integrals.

$6.2 \ell_{1}$-clustering of fully truncated correlation functions. For the KMS state $\langle\cdot\rangle_{\beta, \lambda}$ we consider the fully truncated correlation functions denoted by $\left\langle\prod_{j=1}^{n} a\left(x_{j}, \sigma_{j}\right)\right\rangle_{\beta, \lambda}^{T}$. We refer to Appendix A for their definition. They vanish whenever $\sum_{j=1}^{n} \sigma_{j} \neq 0$ and, as proved in Appendix A, for $n \geq 4$ they do not depend on the operator ordering except for an overall sign. 
Assumption A2 ( $\ell_{1}$-clustering). Let $\beta>0$ and $\mu$ satisfy (6.1). There exists $\lambda_{0}>0$ and $c_{0}>0$ independent of $n$ such that for $0<\lambda \leq \lambda_{0}$. and all $n \geq 4$ one has the bound

$$
\sum_{x \in\left(\mathbb{Z}^{d}\right)^{n}} \delta_{x_{1} 0}\left|\left\langle\prod_{j=1}^{n} a\left(x_{j}, \sigma_{j}\right)\right\rangle_{\beta, \lambda}^{T}\right| \leq \lambda\left(c_{0}\right)^{n} n !
$$

In addition,

$$
\sum_{x \in \mathbb{Z}^{d}}\left|\left\langle a(0)^{*} a(x)\right\rangle_{\beta, \lambda}-\left\langle a(0)^{*} a(x)\right\rangle_{\beta}\right| \leq \lambda 2\left(c_{0}\right)^{2} .
$$

Ginibre [17] studies $\ell_{1}$-clustering for low density quantum gases in the continuum, i.e., for position space $\mathbb{R}^{3}$ and dispersion relation $\omega(k)=k^{2}$. It is rather likely that his analysis could be carried through also for lattice gases. The $L^{1}$-bound by Ginibre is based on an expansion with respect to the fugacity, hence the small coupling regime is not optimally covered. In particular, the prefactor $\lambda$ in (6.19) cannot be deduced from [17]. It seems that extra work is needed.

6.3 Oscillatory integrals. The $\ell_{3}$-dispersivity has been stated already in Assumption A1. In addition we need an assumption which controls the constructive interference between two frequencies.

Assumption A3 (constructive interference). There exists a set $M^{\text {sing }} \subset \mathbb{T}^{d}$ consisting of a union of a finite number of closed, one-dimensional, smooth submanifolds, and a constant $C$ such that for all $t \in \mathbb{R}, k_{0} \in \mathbb{T}^{d}$, and $\sigma \in\{ \pm 1\}$,

$$
\left|\int_{\mathbb{T}^{d}} d k \mathrm{e}^{-\mathrm{i} t\left(\omega(k)+\sigma \omega\left(k-k_{0}\right)\right)}\right| \leq \frac{C\langle t\rangle^{-1}}{d\left(k_{0}, M^{\text {sing }}\right)},
$$

where $d\left(k_{0}, M^{\text {sing }}\right)$ is the distance of $k_{0}$ from $M^{\text {sing }}$.

Remark 6.1 (Dimension). Assumption A3 allows us to cut out a small tube around each curve. If one would cut out too much, e.g. two-dimensional surfaces, this will show up in other parts of the proof. For this reason Assumption A3 as stated requires in addition $d \geq 4$. If 6.21 would hold for $M^{\text {sing }}$ merely a collection of a finite number of points, then we could accommodate $d \geq 3$.

Next we need a mechanism which allows to distinguish between leading and subleading diagrams. For the linear Schrödinger equation with a random potential, this mechanism has been identified in [24]. In the graphical representation developed in this article, it corresponds to the crossing of two edges. Our condition is the natural generalization of the crossing estimate in [24].

Assumption A4 (crossing bounds). Define for $t_{0}, t_{1}, t_{2} \in \mathbb{R}, u_{1}, u_{2} \in \mathbb{T}^{d}$, and $x \in \mathbb{Z}^{d}$,

$$
K\left(x ; t_{0}, t_{1}, t_{2}, u_{1}, u_{2}\right)=\int_{\mathbb{T}^{d}} d k \mathrm{e}^{\mathrm{i} 2 \pi x \cdot k} \mathrm{e}^{-\mathrm{i}\left(t_{0} \omega(k)+t_{1} \omega\left(k+u_{1}\right)+t_{2} \omega\left(k+u_{2}\right)\right)} .
$$

We assume that there is a measurable function $F^{\mathrm{cr}}: \mathbb{T}^{d} \times \mathbb{R}_{+} \rightarrow[0, \infty]$ so that constants $0<\gamma \leq 1, c_{1}, c_{2}$ for the following bounds can be found. 
(i) For any $u_{i} \in \mathbb{T}^{d}, \sigma_{i} \in\{ \pm 1\}, i=1,2,3$, and $0<\beta \leq 1$, the following bounds are satisfied:

$$
\begin{gathered}
\int_{\mathbb{R}^{2}} d s_{1} d s_{2} \mathrm{e}^{-\beta\left(\left|s_{1}\right|+\left|s_{2}\right|\right)}\left\|K\left(s_{1}+s_{2}, \sigma_{1} s_{2}, \sigma_{2} s_{2}, u_{1}, u_{2}\right)\right\|_{3}\left\|p_{s_{1}+s_{2}}\right\|_{3}^{2} \\
\leq \beta^{\gamma-1} F^{\mathrm{cr}}\left(u_{2}-u_{1} ; \beta\right), \\
\int_{\mathbb{R}^{2}} d s_{1} d s_{2} \mathrm{e}^{-\beta\left(\left|s_{1}\right|+\left|s_{2}\right|\right)} \prod_{i=1}^{3}\left\|K\left(s_{1}+s_{2}, \sigma_{i} s_{2}, 0, u_{i}, 0\right)\right\|_{3} \\
\leq \beta^{\gamma-1} F^{\mathrm{cr}}\left(u_{n} ; \beta\right), \quad \text { for any } n \in\{1,2,3\} .
\end{gathered}
$$

(ii) For all $0<\zeta \leq \zeta_{0}$ we have

$$
\int_{\mathbb{T}^{d}} d k F^{\mathrm{cr}}(k ; \zeta) \leq c_{1}\langle\ln \zeta\rangle^{c_{2}}
$$

and if also $u, k_{0} \in \mathbb{T}^{d}, \alpha \in \mathbb{R}, \sigma \in\{ \pm 1\}$, and $n \in\{1,2,3\}$, and we denote $k=$ $\left(k_{1}, k_{2}, k_{0}-k_{1}-k_{2}\right)$, then

$$
\int_{\left(\mathbb{T}^{d}\right)^{2}} d k_{1} d k_{2} F^{\mathrm{cr}}\left(k_{n}+u ; \zeta\right) \frac{1}{|\alpha-\Omega(k, \sigma)+\mathrm{i} \zeta|} \leq c_{1}\langle\ln \zeta\rangle^{1+c_{2}}
$$

where $\Omega:\left(\mathbb{T}^{d}\right)^{3} \times\{ \pm 1\} \rightarrow \mathbb{R}$ is defined by

$$
\Omega(k, \sigma)=\omega\left(k_{3}\right)-\omega\left(k_{1}\right)+\sigma\left(\omega\left(k_{2}\right)-\omega\left(k_{1}+k_{2}+k_{3}\right)\right) .
$$

Remark 6.2 (Reduction). The reader may wonder whether the stated assumptions can be proved for a specified class of $\omega$ 's. Let us first emphasize that the reduction of the many, very high-dimensional oscillatory integrals to a few low dimensional oscillatory integrals involving only $\omega$ is already a big step in the right direction. It is also clear that the reduction cannot be pushed any further. The $\ell_{3}$ dispersivity is needed already to define the limit equation. Constructive interference occurs always at $k_{0}=0$. Thus some control of the phenomenon is required. The crossing bound reflects the mechanism how only a few diagrams survive in the limit. At present, A4 is one version which works, but its optimal form could eventually look differently. In any case, Assumptions A1, A3, and A4 rely on results from a disjoint mathematical discipline. In this sense, the situation is similar to A2. Clustering is proved within rigorous statistical mechanics. In our context it is a necessary input stated in a form which is regarded as obvious by the experts. A1 can be proved by stationary phase methods and holds generically for dimension $d \geq 3$. We are not aware that A3 has ever been studied systematically, but hope that our work might serve as a motivation. A4 in the somewhat simpler context of the random Schrödinger equation has been proved for nearest neighbor couplings in [29] and is investigated more systematically in [30, 31].

Remark 6.3 (Example). For on-site and nearest neighbor couplings only, i.e., $\alpha(x)=0$ for $|x|>1, \alpha(x)=\alpha_{1}$ for $|x|=1$, and arbitrary $\alpha(0)$, A1 is proved in [19] for $d \geq 3$ and 
A3, A4 are proved in [15] for $d \geq 4$. The choice of the constants is $\gamma=\frac{4}{7}, c_{2}=0$, and the function $F^{\mathrm{cr}}$ is taken to be

$$
F^{\mathrm{cr}}(u ; \zeta)=C \prod_{\nu=1}^{d} \frac{1}{\left|\sin \left(2 \pi u^{\nu}\right)\right|^{\frac{1}{7}}}
$$

with a certain constant $C$ depending only on $d$ and $\omega$.

6.4 Main result. To formulate our main result it is convenient to integrate the field against a test function $f \in \ell_{2}$. Interpreting $\langle\cdot, \cdot\rangle$ as inner product, we set

$$
\langle f, a\rangle=\sum_{x \in \mathbb{Z}^{d}} f(x)^{*} a(x), \quad\langle f, a\rangle^{*}=\sum_{x \in \mathbb{Z}^{d}} f(x) a(x)^{*}
$$

and correspondingly for our other conventions as $\langle f, a(t)\rangle$ and $\langle f, a(\sigma, t)\rangle$. In particular

$$
\langle\hat{f}, \hat{a}\rangle=\int_{\mathbb{T}^{d}} d k \hat{f}(k)^{*} \hat{a}(k)=\langle f, a\rangle .
$$

Note that by Schwarz inequality for operators and stationarity

$$
\begin{aligned}
\left|\left\langle\left\langle f_{1}, a\right\rangle^{*}\left\langle f_{2}, a(t)\right\rangle\right\rangle_{\beta, \lambda}\right|^{2} & \leq\left\langle\left\langle f_{1}, a\right\rangle^{*}\left\langle f_{1}, a\right\rangle\right\rangle_{\beta, \lambda}\left\langle\left\langle f_{2}, a\right\rangle^{*}\left\langle f_{2}, a\right\rangle\right\rangle_{\beta, \lambda} \\
= & \int_{\mathbb{T}^{d}} d k W_{\beta, \lambda}^{\theta}(k)\left|\hat{f}_{1}(k)\right|^{2} \int_{\mathbb{T}^{d}} d k W_{\beta, \lambda}^{\theta}(k)\left|\hat{f}_{2}(k)\right|^{2} .
\end{aligned}
$$

Hence the quadratic form $\left(f_{1}, f_{2}\right) \mapsto\left\langle\left\langle f_{1}, a\right\rangle^{*}\left\langle f_{2}, a(t)\right\rangle\right\rangle_{\beta, \lambda}$ is uniformly bounded on $\ell_{2} \times \ell_{2}$. Our main result concerns the behavior of this quadratic form on the kinetic time scale.

Theorem 6.4 Let $\langle\cdot\rangle_{\beta, \lambda}$ satisfy Assumption A2, let $\omega$ satisfy Assumptions A1, A3, and A4, and let $d \geq 4$. Then there exists $t_{0}>0$ such that for $0 \leq t<t_{0}$ one has

$$
\lim _{\lambda \rightarrow 0}\left\langle\left\langle\hat{f}_{2}, \hat{a}\right\rangle^{*}\left\langle\exp \left[-\mathrm{i} t \lambda^{-2} \omega^{\lambda}\right] \hat{f_{1}}, \hat{a}\left(\lambda^{-2} t\right)\right\rangle\right\rangle_{\beta, \lambda}=\int_{\mathbb{T}^{d}} d k W_{\beta, \lambda}^{\theta}(k) \hat{f}_{1}(k)^{*} \mathrm{e}^{-\nu(k) t} \hat{f}_{2}(k)
$$

for all $f_{1}, f_{2} \in \ell_{2}\left(\mathbb{Z}^{d}\right)$.

Remark 6.5 (Short kinetic time). The restriction to a short kinetic time, $t<t_{0}$, has no physical significance. The decay is expected to hold for arbitrary $t_{0}$ and most likely for even longer times. The smallness of $t_{0}$ merely reflects that in our context it is difficult to properly bound the error terms. Technically it arises because in the error term one obtains high order diagrams which are close to the spatially homogeneous case as studied in Section 5, One needs that the leading part of the main term is small. Since the leading diagrams are bounded as $\left(t / t_{0}\right)^{N}$, one has to require $t<t_{0}$.

6.5 Link to the nonlinear Schrödinger equation as discussed in [15]. [15] is written in such a way that the operator ordering, as of relevance in our context, is already respected. 
Thus, with the proper reinterpretation, most formulas hold also for the quantum evolution. In particular, the analysis of the Feynman diagrams carries over verbatim. The Duhamel expansion (2.28) is preliminary and one still needs three modifications to reach the starting point of [15].

- partial time integration. This refers to the time integrations and thus remains valid in the quantum mechanical context.

- insertion of the cutoff functions $\Phi_{0}^{\lambda}, \Phi_{1}^{\lambda}$. This refers to the $k$-integrations and thus remains valid in the quantum mechanical context.

- removal of fast oscillations. As argued above the dispersion relation $\omega$ is renormalized to $\omega^{\lambda}$. Thus, as a modification of (2.19), we define

$$
\hat{a}(k, 1, t)=e^{\mathrm{i} \omega^{\lambda}(k) t} \hat{a}(k, t), \quad \hat{a}(k,-1, t)=e^{-\mathrm{i} \omega^{\lambda}(k) t} \hat{a}(-k, t)^{*} .
$$

In addition we introduce the pair truncation

$$
\begin{aligned}
\hat{\mathcal{P}}\left(\hat{a}\left(k_{1},-1\right) \hat{a}\left(k_{2}, \sigma\right) \hat{a}\left(k_{3}, 1\right)\right) & \\
= & \hat{a}\left(k_{1},-1\right) \hat{a}\left(k_{2}, \sigma\right) \hat{a}\left(k_{3}, 1\right)-\left(\left\langle\hat{a}\left(k_{1},-1\right) \hat{a}\left(k_{2}, \sigma\right)\right\rangle_{\beta, \lambda} \hat{a}\left(k_{3}, 1\right)\right. \\
& \left.\quad+\theta\left\langle\hat{a}\left(k_{1},-1\right) \hat{a}\left(k_{3}, 1\right)\right\rangle_{\beta, \lambda} \hat{a}\left(k_{2}, \sigma\right)+\left\langle\hat{a}\left(k_{2}, \sigma\right) \hat{a}\left(k_{3}, 1\right)\right\rangle_{\beta, \lambda} \hat{a}\left(k_{1},-1\right)\right),
\end{aligned}
$$

$\sigma= \pm 1$ and omitting the $t$ argument. Then $\hat{a}\left(k_{1}, \sigma, t\right)$ satisfies the evolution equation

$$
\begin{aligned}
\frac{d}{d t} \hat{a}\left(k_{1}, \sigma, t\right)= & -\mathrm{i} \lambda \sigma \int_{\left(\mathbb{T}^{d}\right)^{3}} d k_{2} d k_{3} d k_{4} \delta\left(k_{1}-k_{2}-k_{3}-k_{4}\right) \\
& \times \frac{1}{2}\left((1+\sigma) \widehat{V}\left(k_{2}+k_{3}\right)+(1-\sigma) \widehat{V}\left(k_{3}+k_{4}\right)\right) \\
& \times \mathrm{e}^{-\mathrm{i} t \Omega(k, \sigma)}\left\{\Phi_{1}^{\lambda}\left(k_{2}, k_{3}, k_{4}\right) \hat{a}\left(k_{2},-1, t\right) \hat{a}\left(k_{3}, \sigma, t\right) \hat{a}\left(k_{4}, 1, t\right)\right. \\
& \left.+\Phi_{0}^{\lambda}\left(k_{2}, k_{3}, k_{4}\right) \hat{\mathcal{P}}\left[\hat{a}\left(k_{2},-1, t\right) \hat{a}\left(k_{3}, \sigma, t\right) \hat{a}\left(k_{4}, 1, t\right)\right]\right\},
\end{aligned}
$$

where

$$
\Omega(k, \sigma)=-\sigma \omega^{\lambda}\left(k_{1}\right)-\omega^{\lambda}\left(k_{2}\right)+\sigma \omega^{\lambda}\left(k_{3}\right)+\omega^{\lambda}\left(k_{4}\right) .
$$

(6.35) agrees with the corresponding formula of [15] with the only modification consisting of a general interaction potential. In [15] we consider the case $\hat{V}(k)=\hat{V}(0)$. Therefore the factor in (6.35) containing $\widehat{V}$ is replaced by a constant and $\omega^{\lambda}(k)=\omega(k)+\lambda R_{0}$, where $R_{0}$ depends on $\lambda$ but not on $k$. In frequency differences $R_{0}$ thus drops out.

We are confident that the estimates of the oscillatory integrals proved in [15] still hold for the case under consideration here. Of course, a simple estimate as

$$
\left|\mathrm{e}^{-\mathrm{i} \omega(k) t}-\mathrm{e}^{-\mathrm{i} \omega^{\lambda}(k) t}\right| \leq C \lambda|t|
$$

uniformly in $k$ is too crude. Rather each oscillating integral has to be estimated with an $\omega^{\lambda}$ depending weakly on $\lambda$. This step remains to be carried out.

The classical average $\mathbb{E}$ in [15] becomes the quantum average $\langle\cdot\rangle_{\beta, \lambda}$ over the KMS state. With the assumption A2, higher cumulants do not contribute. For the quasifree 
part one has either $W$ or $\tilde{W}$ as time $t=0$ input. Since both functions are smooth, the estimates from the commutative case remain valid.

6.6 Error terms. We adopt the notation from [15]. There are three error terms of the same structure, namely

$$
\left\langle\left\langle\hat{f}_{1}, \hat{a}(0)\right\rangle^{*} \int_{0}^{t} d s\left\langle\hat{f}_{2}, \mathcal{X}_{n}(t, s)[\hat{a}(s)]\right\rangle\right\rangle_{\beta, \lambda}
$$

with $\mathcal{X}$ any of $\mathcal{G}, \mathcal{Z}$, or $\mathcal{A}$. We use the Schwarz inequality for operators $A, B$ according to which

$$
\left.\left|\left\langle A^{*} B\right\rangle_{\beta, \lambda}\right|^{2} \leq\left.\left\langle|A|^{2}\right\rangle_{\beta, \lambda}\langle] B\right|^{2}\right\rangle_{\beta, \lambda}
$$

with the shorthand $A^{*} A=|A|^{2}$. Then

$$
\begin{aligned}
& \left|\left\langle\left\langle\hat{f}_{1}, \hat{a}(0)\right\rangle^{*}\left\langle\hat{f}_{2}, \int_{0}^{t} d s \mathcal{X}_{n}(s, t)[\hat{a}(s)]\right\rangle\right\rangle_{\beta, \lambda}\right|^{2} \\
& \quad \leq\left(\int_{0}^{t} d s\left|\left\langle\left\langle\hat{f}_{1}, \hat{a}(0)\right\rangle\left\langle\hat{f}_{2}, \mathcal{X}_{n}(s, t)[\hat{a}(s)]\right\rangle\right\rangle_{\beta, \lambda}\right|\right)^{2} \\
& \quad \leq t\left\langle\left|\left\langle\hat{f}_{1}, \hat{a}(0)\right\rangle\right|^{2}\right\rangle_{\beta, \lambda} \int_{0}^{t} d s\left\langle\left|\left\langle\hat{f}_{2}, \mathcal{X}_{n}(t, s)[\hat{a}(s)]\right\rangle\right|^{2}\right\rangle_{\beta, \lambda} .
\end{aligned}
$$

$\mathcal{X}_{n}(t, s)$ is a monomial of order $n$ in the factors $a(k, \sigma, s)$ which differ by a phase factor from $a(k, s), a(k, s)^{*}$. The latter field operators are invariant for $\langle\cdot\rangle_{\beta, \lambda}$ and, at the expense of a phase factor, $\hat{a}(s)$ in (6.40) can be replaced by $\hat{a}(0)$. Thus, at given cutoff $N$, the two-point function of (6.32) is reduced to a main term and an error term, which both involve only the free time evolution and are monomials of the time 0 fields $\hat{a}$ averaged with respect to the $\beta$-KMS state $\langle\cdot\rangle_{\beta, \lambda}$.

6.7 Convergence of the leading part of the main term. We refer to Section 5 for the general structure. For the case under consideration the Feynman diagrams are constrained. In (6.32) the oscillating term is combined with $\left\langle\hat{f}_{2}, \hat{a}\right\rangle^{*}$. The Feynman diagram has one line segment $[0, t]$ with parity -1 , which is constrained not to fuse at all. In the time slice $\left[t-s_{n}, t\right]$ there is only one further line segment. Thus $n_{0}=2$. A leading diagram is still defined by the pairing property for even time slices and the absence of the factor $\widehat{V}(0)$. The line corresponding to $\left\langle\hat{f}_{2}, \hat{a}\right\rangle^{*}$ has momentum $k_{1}$. The collision operator acting on $k_{2}, \ldots, k_{n+2}$ is defined as in (5.18). Because of the constraint the collision operator acting on $k_{1}$ reads

$$
\begin{aligned}
\left(\mathcal{C}_{1, n+2}^{-}\right. & \left.(t) \widehat{\rho}_{n+2}\right)\left(k_{1}, \tau_{1}, \ldots, k_{n}, \tau_{n}\right)=2 \int_{\left(\mathbb{T}^{d}\right)^{3}} d g_{2} d g_{3} d g_{4} \delta\left(k_{1}+g_{2}-g_{3}-g_{4}\right) \\
& \times \mathrm{e}^{\mathrm{i} t\left(\omega\left(k_{1}\right)+\omega\left(g_{2}\right)-\omega\left(g_{3}\right)-\omega\left(g_{4}\right)\right)} \widehat{V}\left(k_{2}-k_{3}\right)\left(\widehat{V}\left(k_{2}-k_{3}\right)+\theta \widehat{V}\left(k_{2}-k_{4}\right)\right) \\
& \times\left(\theta \widehat{\rho}_{n+2}\left(k_{1}, \tau_{1}, \ldots, g_{3}, 1, g_{4}, 1\right)-\widehat{\rho}_{n+1}\left(k_{1}, \tau_{1}, \ldots, g_{2}, 1, g_{3},-1\right)\right. \\
& \left.\quad-\theta \widehat{\rho}_{n+2}\left(k_{1}, \tau_{1}, \ldots, g_{2}, 1, g_{4}, 1\right)\right) .
\end{aligned}
$$

The initial conditions are

$$
\widehat{\rho}_{n}\left(k_{1}, \tau_{1}, \ldots, k_{n}, \tau_{n}\right)=\prod_{j=1}^{n} W_{\beta}\left(k_{j}, \tau_{j}\right) .
$$


We set

$$
\mathcal{C}_{n+2}^{-}(t)=\mathcal{C}_{1, n+2}^{-}(t)+\sum_{j=2}^{n} \mathcal{C}_{j, n+2}(t)
$$

Let us define $\mathcal{M}_{n}^{\lambda}(t)$ as the sum of all leading Feynman diagrams at order $n$. With the above notation it is given by

$$
\begin{aligned}
\mathcal{M}_{n}^{\lambda}(t)= & \lambda^{2 n} \int_{\left(\mathbb{R}_{+}\right)^{n+1}} d \underline{t} \int_{\left(\mathbb{R}_{+}\right)^{n}} d \underline{s} \delta\left(\sum_{j=1}^{n+1} t_{j}+\sum_{j=1}^{n} s_{j}-t\right) \int_{\mathbb{T}^{d}} d k_{1} \hat{f}_{1}\left(k_{1}\right)^{*} \hat{f}_{2}\left(k_{1}\right) \\
& \left(\mathcal{C}_{3}^{-}\left(s_{1}\right) \ldots \mathcal{C}_{2 n+1}^{-}\left(s_{n}\right) \widehat{\rho}_{2 n+1}\right)\left(k_{1}, 1\right) .
\end{aligned}
$$

Proposition 5.7 remains valid. Thus one can pass to the limit as

$$
\lim _{\lambda \rightarrow 0} \mathcal{M}_{n}^{\lambda}\left(\lambda^{-2} t\right)=\frac{1}{n !} t^{n} \int_{\mathbb{T}^{d}} d k_{1} \hat{f}_{1}\left(k_{1}\right)^{*} \hat{f}_{2}\left(k_{1}\right)\left(\mathcal{C}_{3}^{-} \ldots \mathcal{C}_{2 n+1}^{-} \widehat{\rho}_{2 n+1}\right)\left(k_{1}, 1\right)
$$

Let $\widehat{\rho}_{3}$ be given by (6.42). Then

$$
\begin{aligned}
\left(\mathcal{C}_{1,3}(s) \widehat{\rho}_{3}\right)\left(k_{1}, \tau_{1}\right) & =\frac{d}{d s} \int_{\left(\mathbb{T}^{d}\right)^{3}} d k_{2} d k_{3} d k_{4} \delta\left(k_{1}+k_{2}-k_{3}-k_{4}\right) \\
& \times(\sin \Omega s) \Omega^{-1}\left(\mathrm{e}^{\beta \Omega}-1\right)\left(\widehat{V}\left(k_{2}-k_{3}\right)+\theta\left(\widehat{V}\left(k_{2}-k_{4}\right)\right)^{2}\right. \\
& \times W_{\beta}\left(k_{1}, \tau_{1}\right) W_{\beta}\left(k_{2}, \tau_{1}\right) W_{\beta}\left(k_{3},-\tau_{1}\right) W_{\beta}\left(k_{4},-\tau_{1}\right)
\end{aligned}
$$

with $\Omega=\omega_{1}+\omega_{2}-\omega_{3}-\omega_{4}$. Therefore

$$
\int_{0}^{\infty} d s\left(\mathcal{C}_{1,3}(s) \widehat{\rho}_{3}\right)\left(k_{1}, \tau_{1}\right)=0
$$

Returning to 6.45) we conclude that when acting with $\mathcal{C}_{2 j+1}^{-}$, where $j=1, \ldots, n$, only its first summand, i.e., $\mathcal{C}_{1,2 j+1}^{-}$, contributes. Hence, there exists $t_{0}$ such that for $0 \leq t \leq t_{0}$ one has

$$
\lim _{\lambda \rightarrow 0} \sum_{n=0}^{\infty} \mathcal{M}_{n}^{\lambda}\left(\lambda^{-2} t\right)=\sum_{n=0}^{\infty} \frac{t^{n}}{n !}(-1)^{n} \int_{\mathbb{T}^{d}} d k \hat{f}_{1}(k)^{*} \hat{f}_{2}(k) W_{\beta}^{\theta}(k) \nu(k)^{n}
$$

Remark 6.6 (Radius of convergence). The integral in (6.46) decays at least as $s^{-\delta}$. Therefore the error in (6.47) is order $\lambda^{2 \delta}$. The leading diagrams at order $n$ have a single term which yields the non-zero limit in (6.48), while the remaining $(n !-1)$ terms are of order $\lambda^{2 \delta}$. Therefore one can cut the series at order $N$ with $\lambda^{2 \delta} N !=1$. Since the series of contributing terms has an infinite radius of convergence, the restriction to bounded $t_{0}$ can be lifted and (6.48) holds in fact for all $t$. On the other hand the error term can be controlled only for $|t| \leq t_{0}$ and the extra effort will not improve Theorem 6.3. 


\section{Time correlations for the number density}

Physically of great interest are the density current and energy current time correlations. They differ in two respects from the correlation function studied in the previous section: they are four-point functions and, more importantly, involve a spatial summation. Slightly formal, these correlation functions are particular cases of the number density time correlations in equilibrium, i.e., of $\left\langle\hat{a}(k, t)^{*} \hat{a}(k, t) \hat{a}\left(k^{\prime}, 0\right)^{*} \hat{a}\left(k^{\prime}, 0\right)\right\rangle_{\beta, \lambda}$.

Let $\eta: \mathbb{Z}^{d} \rightarrow \mathbb{C}$ such that $\eta(x)^{*}=\eta(-x)$ and $\eta$ has bounded support. We define the energy-like, resp. number-like, observable

$$
H^{\eta}=\sum_{x, y \in \mathbb{Z}^{d}} \eta(x-y) a(x)^{*} a(y)
$$

and represent it by a sum of local terms as

$$
\begin{aligned}
& H^{\eta}=\sum_{w \in \mathbb{Z}^{d}} H_{w}^{\eta} \\
& H_{w}^{\eta}=\frac{1}{2} \sum_{y \in \mathbb{Z}^{d}}\left(a(w)^{*} \eta(w-y) a(y)+a(y)^{*} \eta(y-w) a(w)\right) .
\end{aligned}
$$

For $H^{\eta}=H_{\text {har }}$ one has $\hat{\eta}(k)=\omega(k)$, for the energy current $\hat{\eta}(k)=\left(\nabla_{k} \omega(k)\right) \omega(k)$, and, correspondingly, $\hat{\eta}(k)=1, \hat{\eta}(k)=\nabla_{k} \omega(k)$ for number and number current.

As before $\langle\cdot\rangle_{\beta, \lambda}$ is the $\beta$-KMS state for $H-\mu N$ at infinite volume with $\mu$ satisfying (6.1) and we set $\langle\cdot\rangle_{\beta, 0}=\langle\cdot\rangle_{\beta}$. It is also convenient to introduce the Kubo inner product defined through

$$
\langle\langle A, B\rangle\rangle_{\beta, \lambda}=\beta^{-1} \int_{0}^{\beta} d \beta^{\prime}\left(\left\langle A^{*} e^{-\beta^{\prime} H} B e^{\beta^{\prime} H}\right\rangle_{\beta, \lambda}-\left\langle A^{*}\right\rangle_{\beta, \lambda}\langle B\rangle_{\beta, \lambda}\right) .
$$

The fluctuations of $H^{\eta}$ in a large box $\Lambda$ are given by

$$
\xi_{\Lambda}^{\eta}=|\Lambda|^{-1 / 2} \sum_{w \in \Lambda}\left(H_{w}^{\eta}-\left\langle H_{w}^{\eta}\right\rangle_{\beta, \lambda}\right)
$$

and the quantity of interest is the time-displaced covariance of (7.4), which reads

$$
\lim _{\Lambda \uparrow \mathbb{Z}^{d}}\left\langle\left\langle\xi_{\Lambda}^{\eta}, \xi_{\Lambda}^{\eta}(t)\right\rangle\right\rangle_{\beta, \lambda}=\sum_{w \in \mathbb{Z}^{d}}\left\langle\left\langle H_{w}^{\eta}, H_{0}^{\eta}(t)\right\rangle\right\rangle_{\beta, \lambda}=C_{\lambda}^{\eta}(t)
$$

In particular, at $t=0$,

$$
\lim _{\lambda \rightarrow 0} \lim _{\Lambda \uparrow \mathbb{Z}^{d}}\left\langle\left\langle\xi_{\Lambda}^{\eta}, \xi_{\Lambda}^{\eta}\right\rangle\right\rangle_{\beta, \lambda}=\sum_{w \in \mathbb{Z}^{d}}\left\langle\left\langle H_{w}^{\eta}, H_{0}^{\eta}\right\rangle\right\rangle_{\beta}=\int_{\mathbb{T}^{d}} d k|\hat{\eta}(k)|^{2} W_{\beta}^{\theta}(k) \tilde{W}_{\beta}^{\theta}(k) .
$$

Note that in the limit $\lambda \rightarrow 0$ the Kubo and standard inner product coincide for the observables under consideration. 
As before the kinetic limit provides information on $C_{\lambda}^{\eta}\left(\lambda^{-2} t\right)$ for small $\lambda$. There is a simple formal argument how to guess this correlation by building on the results from Section 5, Let $\langle\cdot\rangle_{\beta, \lambda}(\varepsilon)$ be the $\beta$-KMS state for $H-\mu N+(\varepsilon / \beta) H^{\eta}$. Then

$$
C_{\lambda}^{\eta}(t)=\left.\frac{\partial}{\partial \varepsilon}\left\langle H_{0}^{\eta}(t)\right\rangle_{\beta, \lambda}(\varepsilon)\right|_{\varepsilon=0} .
$$

We now perform first the limit $\lambda \rightarrow 0$ and then the limit $\varepsilon \rightarrow 0$. For time $t=0$ one has

$$
\lim _{\lambda \rightarrow 0}\left\langle\hat{a}(k)^{*} \hat{a}\left(k^{\prime}\right)\right\rangle_{\beta, \lambda}(\varepsilon)=\delta\left(k-k^{\prime}\right) W^{\varepsilon}(k),
$$

where

$$
W^{\varepsilon}(k)=\left(\mathrm{e}^{[\beta(\omega(k)-\mu)+\varepsilon \hat{\eta}(k)]}-\theta\right)^{-1},
$$

which is well-defined for $\varepsilon$ sufficiently small. Now, by Section 5 ,

$$
\lim _{\lambda \rightarrow 0}\left\langle\hat{a}\left(k, \lambda^{-2} t\right)^{*} \hat{a}\left(k^{\prime}, \lambda^{-2} t\right)\right\rangle_{\beta, \lambda}(\varepsilon)=\delta\left(k-k^{\prime}\right) W^{\varepsilon}(k, t),
$$

and $W^{\varepsilon}(k, t)$ solves (5.4) with initial condition $W^{\varepsilon}(k)$. It follows that

$$
\lim _{\lambda \rightarrow 0}\left\langle H_{0}^{\eta}\left(\lambda^{-2} t\right)\right\rangle_{\beta, \lambda}(\varepsilon)=\int_{\mathbb{T}^{d}} d k \hat{\eta}(k) W^{\varepsilon}(k, t) .
$$

We linearize the collision operator as

$$
\mathcal{C}\left(W_{\beta}^{\theta}+\varepsilon f\right)=\varepsilon A f+\mathcal{O}\left(\varepsilon^{2}\right) .
$$

It is convenient to introduce the multiplication operator $U_{\beta}$ through

$$
\left(U_{\beta} f\right)(k)=\left(W_{\beta}^{\theta}(k) \tilde{W}_{\beta}^{\theta}(k)\right)^{1 / 2} f(k),
$$

and to define

$$
L f=-A U_{\beta}^{2} f .
$$

Expanding (7.11),

$$
\left.\frac{\partial}{\partial \varepsilon} \int_{\mathbb{T}^{d}} d k \hat{\eta}(k) W^{\varepsilon}(k, t)\right|_{\varepsilon=0}=\left\langle\hat{\eta}, \mathrm{e}^{A t} U_{\beta}^{2} \hat{\eta}\right\rangle,
$$

where $\langle\cdot, \cdot\rangle$ denotes now the inner product in $L^{2}\left(\mathbb{T}^{d}, d k\right)$. Using the definition of $L,(7.15)$ can be written in the more symmetric form as

$$
\left\langle\hat{\eta}, \mathrm{e}^{A t} U_{\beta}^{2} \widehat{\eta}\right\rangle=\left\langle U_{\beta} \hat{\eta}, \exp \left[-U_{\beta}^{-1} L U_{\beta}^{-1} t\right] U_{\beta} \hat{\eta}\right\rangle
$$

From the linearization one obtains

$$
\begin{aligned}
(L f)\left(k_{1}\right) & =\pi \int_{\left(\mathbb{T}^{d}\right)^{3}} d k_{2} d k_{3} d k_{4} \delta\left(k_{1}+k_{2}-k_{3}-k_{4}\right) \delta\left(\omega_{1}+\omega_{2}-\omega_{3}-\omega_{4}\right) \\
\times & \widehat{V}\left(k_{2}-k_{3}\right)+\left.\theta \widehat{V}\left(k_{2}-k_{4}\right)\right|^{2} W_{\beta, 1}^{\theta} W_{\beta, 2}^{\theta} \tilde{W}_{\beta, 3}^{\theta} \tilde{W}_{\beta, 4}^{\theta}\left(f_{1}+f_{2}-f_{3}-f_{4}\right) .
\end{aligned}
$$


The quadratic form associated to $L$ reads then

$$
\begin{aligned}
\langle f, L f\rangle & =\frac{\pi}{4} \int_{\left(\mathbb{T}^{d}\right)^{4}} d k_{1} d k_{2} d k_{3} d k_{4} \delta\left(k_{1}+k_{2}-k_{3}-k_{4}\right) \delta\left(\omega_{1}+\omega_{2}-\omega_{3}-\omega_{4}\right) \\
\times & \times \widehat{V}\left(k_{2}-k_{3}\right)+\left.\theta \widehat{V}\left(k_{2}-k_{4}\right)\right|^{2} W_{\beta, 1}^{\theta} W_{\beta, 2}^{\theta} \tilde{W}_{\beta, 3}^{\theta} \tilde{W}_{\beta, 4}^{\theta}\left(f_{1}+f_{2}-f_{3}-f_{4}\right)^{2} .
\end{aligned}
$$

Therefore $L=L^{*}$ and $L \geq 0$. Clearly $L 1=0$ and $L \omega=0$. Thus the zero subspace is at least two-fold degenerate. If the $\mathrm{H}$-theorem holds, see Appendix B, then the 0 eigenvalue of $L$ is exactly two-fold degenerate. In brackets, we note that momentum conservation is destroyed by the underlying lattice. More precisely, if one sets $f(k)=k \bmod 1$, then $L f \neq 0$, since the umklapp $k_{1}+k_{2}-k_{3}-k_{4}=n, n$ integer vector and $n \neq 0$, is permitted according to the momentum $\delta$-function.

Allowing for the interchange of limits we arrive at

Conjecture 7.1 Under suitable conditions on $\omega$ it holds

$$
\lim _{\lambda \rightarrow 0} C_{\lambda}^{\eta}\left(\lambda^{-2} t\right)=\left\langle U_{\beta} \hat{\eta}, \exp \left[-U_{\beta}^{-1} L U_{\beta}^{-1}|t|\right] U_{\beta} \hat{\eta}\right\rangle .
$$

Duhamel expansion. We want to explore whether, at least in principle, the Duhamel expansion of Section 2 could work. The starting point is (2.28) for $n_{0}=2$. This results in

$$
H_{0}^{\eta}(t)=H_{0, \text { main }}^{\eta}(t)+H_{0, \text { error }}^{\eta}(t) .
$$

The main term is discussed first.

We insert the main term in the definition of $C_{\lambda}^{\eta}(t)$. Then the time $t=0$ input for the Feynman diagrams is of the generic form

$$
\sum_{w \in \mathbb{Z}^{d}}\left\langle\left\langle H_{w}^{\eta}, \prod_{j=1}^{n} \hat{a}\left(k_{j}, \sigma_{j}\right)\right\rangle\right\rangle_{\beta, \lambda}=\left.\frac{\partial}{\partial \varepsilon}\left\langle\prod_{j=1}^{n} \hat{a}\left(k_{j}, \sigma_{j}\right)\right\rangle_{\beta, \lambda}(\varepsilon)\right|_{\varepsilon=0} .
$$

Therefore we have to require a slightly modified $\ell_{1}$-clustering as

Assumption A5. (strengthened $\ell_{1}$-clustering). Let $\beta>0$ and $\mu$ satisfy (6.1). There exists $\lambda_{0}>0$ and $c_{0}>0$ independent of $n$ such that for $0<\lambda \leq \lambda_{0}$ and all $n \geq 4$ one has the bound

$$
\sum_{x \in\left(\mathbb{Z}^{d}\right)^{n}} \delta_{x_{1} 0}\left|\frac{\partial}{\partial \varepsilon}\left\langle\prod_{j=1}^{n} a\left(x_{j}, \sigma_{j}\right)\right\rangle_{\beta, \lambda}(\varepsilon)\right|_{\varepsilon=0} \mid \leq \lambda\left(c_{0}\right)^{n} n ! .
$$

In addition the following limit exists uniformly in $k$

$$
\lim _{\lambda \rightarrow 0} \sum_{x \in \mathbb{Z}}\left|\frac{\partial}{\partial \varepsilon}\left\langle a(x)^{*} a(0)\right\rangle_{\beta, \lambda}(\varepsilon)\right|_{\varepsilon=0}-\left(U_{\beta}^{2} \hat{\eta}\right)^{\curlyvee}(x) \mid=0 .
$$

Under Assumption A5, the input function for the Feynman diagrams has the same regularity as used in Section 6 . We conclude that together with Assumptions A1, A3, and A4, the Duhamel expansion converges term to the conjectured limit (7.19). 
For the error term one has symbolically

$$
H_{w, \text { error }}^{\eta}(t)=\int_{0}^{t} d s \mathcal{F}_{N, w}^{\eta}(t-s)[\hat{a}(s)] .
$$

For the Kubo inner product we use Schwarz inequality as in (6.39) to conclude

$$
\begin{aligned}
& \left(\sum_{w \in \mathbb{Z}^{d}}\left\langle\left\langle H_{w}^{\eta}, H_{0, \text { error }}^{\eta}(t)\right\rangle\right\rangle_{\beta, \lambda}\right)^{2} \\
& \leq t\left(\sum_{w \in \mathbb{Z}^{d}}\left\langle\left\langle H_{0}^{\eta}, H_{w}^{\eta}\right\rangle\right\rangle_{\beta, \lambda}\right) \int_{0}^{t} d s \sum_{w \in \mathbb{Z}^{d}}\left\langle\left\langle\mathcal{F}_{N, 0}^{\eta}(t-s)[\hat{a}(s)], \mathcal{F}_{N, w}^{\eta}(t-s)[\hat{a}(s)]\right\rangle\right\rangle_{\beta, \lambda} \cdot(7.2
\end{aligned}
$$

The first factor is bounded. In the second factor we use stationary to arrive at

$$
\int_{0}^{t} d s \sum_{w \in \mathbb{Z}^{d}}\left\langle\left\langle\mathcal{F}_{N, 0}(s)[\hat{a}], \mathcal{F}_{N, w}(s)[\hat{a}]\right\rangle\right\rangle_{\beta, \lambda} .
$$

Proceeding as before, one would like to take the $\sum_{w}$ in the exponential to modify the state $\langle\cdot\rangle_{\beta, \lambda}$. But this would be a high order polynomial, hence difficult to control. One may view (7.26) also as Feynman diagrams with singular initial conditions resulting from the one $\delta$-function because of the $\sum_{w}$. This would require to redo the oscillatory integrals. At present it is not clear whether such an approach could work even in principle.

\section{A Appendix. Truncated correlation functions}

We follow Bratelli and Robinson [18], pages 39 and 43.

Let $\langle\cdot\rangle$ be an even state on the CCR, resp. CAR, algebra. The moments are assumed to exist and odd moments vanish. We use $a_{j}$ as shorthand for $\left\langle f_{j}, a_{j}\left(\sigma_{j}\right)\right\rangle$. Then the moments are

$$
\left\langle\prod_{j=1}^{n} a_{j}\right\rangle
$$

and the fully truncated moments are denoted by

$$
\left\langle\prod_{j=1}^{n} a_{j}\right\rangle^{T}
$$

To define them, let $\mathfrak{J}$ denote an index set and $F$ a function from the non empty ordered subsets of $\mathfrak{J}$ to the complex numbers. The truncation $F_{T}$ of $F$ is now given recursively by

$$
F(I)=\sum_{\mathcal{P}_{I}} \varepsilon\left(\mathcal{P}_{I}\right) \prod_{J \in \mathcal{P}_{I}} F_{T}(J),
$$

where the sum is over all partitions $\mathcal{P}_{I}$ of $I$ into ordered even subsets, $\mathcal{P}_{I}=\left\{J_{1}, \ldots, J_{n}\right\}$ and $\varepsilon\left(\mathcal{P}_{I}\right)=1$ for bosons and $\varepsilon\left(\mathcal{P}_{I}\right)= \pm 1$ is according to whether the permutation 
$I \mapsto\left(J_{1}, \ldots, J_{n}\right)$ is even or odd. In our case $F$ are the moments and $F_{T}$ their full truncation.

Theorem A.1 (truncated correlations). Let $n$ be even, $n \geq 4$, and let $\pi$ be a permutation of $I=(1, \ldots, n)$. Then

$$
\left\langle\prod_{j=1}^{n} a_{j}\right\rangle^{T}=\varepsilon(\pi)\left\langle\prod_{j=1}^{n} a_{\pi(j)}\right\rangle^{T},
$$

where $\varepsilon(\pi)=1$ for bosons and for fermions $\varepsilon(\pi)=\operatorname{sign} \pi$, the sign of the permutation $\pi$.

Proof: Let $I=\{1, \ldots, n\}$ and $\alpha=\{m, m+1\} \subset I$. We set

$$
F(I)=\left\langle a_{1} \ldots a_{n}\right\rangle, \quad \widetilde{F}(I)=\left\langle a_{1} \ldots a_{m+1} a_{m} \ldots a_{n}\right\rangle .
$$

It holds

$$
F(I)=\sum_{\alpha \in J \subset I} \varepsilon(J, I \backslash J) F_{T}(J) F(I \backslash J)
$$

where $\varepsilon(J, I \backslash J)=1$ for bosons and $\varepsilon(J, I \backslash J)= \pm 1$ for fermions according to whether $I \mapsto(J, I \backslash J)$ is an even or odd permutation of $I$. Since $a_{m} a_{m+1}-\theta a_{m+1} a_{m}=c \mathbb{1}$, $c \in \mathbb{C}$, one has

$$
\begin{aligned}
& c F(I \backslash \alpha)=F(I)-\theta \widetilde{F}(I)=\sum_{\alpha \in J \subset I} \varepsilon(J, I \backslash J)\left(F_{T}(J)-\theta \widetilde{F}_{T}(J)\right) F(I \backslash J) \quad \text { (A.7) } \\
& =\left(F_{T}(\alpha)-\theta \widetilde{F}_{T}(\alpha)\right) F(I \backslash \alpha)+\sum_{\alpha \in J \subset I, J \neq \alpha} \varepsilon(J, I \backslash J)\left(F_{T}(J)-\theta \widetilde{F}_{T}(J)\right) F(I \backslash J) .
\end{aligned}
$$

Since $F_{T}(\alpha)-\theta \widetilde{F}_{T}(\alpha)=c$, we conclude

$$
0=\sum_{\alpha \in J \subset I, J \neq \alpha} \varepsilon(J, I \backslash J)\left(F_{T}(J)-\theta \widetilde{F}_{T}(J)\right) F(I \backslash J)
$$

For $|I|=4$, the only summand is $J=I$ and $F_{T}(I)=\theta \widetilde{F}_{T}(I)$ since $F(\emptyset)=1$. By iterating (A.8), one concludes the validity of (A.4).

\section{B Appendix. Some properties of the spatially homoge- neous Boltzmann-Nordheim equation}

The purpose of this appendix is to provide a rather compressed list of the basic properties of the spatially homogeneous Boltzmann-Nordheim equation and to point at the relevant literature. It will be convenient to discuss fermions and bosons separately. 


\section{B.1 Fermions}

The Boltzmann-Nordheim equation reads, see (5.4), (5.5),

$$
\begin{gathered}
\frac{\partial}{\partial t} W(k, t)=\mathcal{C}(W(t))(k), \\
\mathcal{C}(W)\left(k_{1}\right)=\pi \int_{\left(\mathbb{T}^{d}\right)^{3}} d k_{2} d k_{3} d k_{4} \delta\left(k_{1}+k_{2}-k_{3}-k_{4}\right) \delta\left(\omega_{1}+\omega_{2}-\omega_{3}-\omega_{4}\right) \\
\left|\widehat{V}\left(k_{2}-k_{3}\right)-\widehat{V}\left(k_{2}-k_{4}\right)\right|^{2}\left(\tilde{W}_{1} \tilde{W}_{2} W_{3} W_{4}-W_{1} W_{2} \tilde{W}_{3} \tilde{W}_{4}\right),
\end{gathered}
$$

$\tilde{W}=1-W$. It has to be solved for initial data $W$ such that $0 \leq W(k) \leq 1$. If there would be a first time $t$ such that $W\left(k_{0}, t\right)=0$, then $\mathcal{C}(W(t))\left(k_{0}\right) \geq 0$, and correspondingly $W\left(k_{1}, t\right)=1$ implies that $\mathcal{C}(W(t))\left(k_{1}\right) \leq 0$. Hence the constraint $0 \leq W \leq 1$ is preserved in time. Note that $\mathcal{C}(W)=0$ in case $\widehat{V}(k)=\widehat{V}(0)$.

At least formally, total number and energy are conserved,

$$
\int_{\mathbb{T}^{d}} d k_{1} \mathcal{C}(W)\left(k_{1}\right)=0, \quad \int_{\mathbb{T}^{d}} d k_{1} \omega\left(k_{1}\right) \mathcal{C}(W)\left(k_{1}\right)=0 .
$$

According to the Fermi statistics, the entropy per volume of a quasifree state is given by

$$
S(W)=-\int_{\mathbb{T}^{d}} d k(W(k) \log W(k)+\tilde{W}(k) \log \tilde{W}(k)) .
$$

The entropy changes in time as

$$
\frac{d}{d t} S(W(t))=\sigma(W(t))
$$

with the entropy production

$$
\begin{gathered}
\sigma(W)=\pi \int_{\left(\mathbb{T}^{d}\right)^{4}} d k_{1} d k_{2} d k_{3} d k_{4} \delta\left(k_{1}+k_{2}-k_{3}-k_{4}\right) \delta\left(\omega_{1}+\omega_{2}-\omega_{3}-\omega_{4}\right) \\
\left|\widehat{V}\left(k_{2}-k_{3}\right)-\widehat{V}\left(k_{2}-k_{4}\right)\right|^{2} F\left(\tilde{W}_{1} \tilde{W}_{2} W_{3} W_{4}, W_{1} W_{2} \tilde{W}_{3} \tilde{W}_{4}\right)
\end{gathered}
$$

and $F(x, y)=(x-y) \log (x / y)$. Hence

$$
\sigma \geq 0
$$

which is the H-theorem. $\sigma=0$ if and only if

$$
\frac{W_{1} W_{2}}{\tilde{W}_{1} \tilde{W}_{2}}=\frac{W_{3} W_{4}}{\tilde{W}_{3} \tilde{W}_{4}}
$$

on the collision set $\mathcal{D}_{\mathrm{c}}=\left\{\left(k_{1}, k_{2}, k_{3}, k_{4}\right) \in \mathbb{R}^{12} \mid k_{1}+k_{2}=k_{3}+k_{4}, \omega_{1}+\omega_{2}=\omega_{3}+\omega_{4}\right\}$. If one introduces

$$
\phi=\log (W / \tilde{W})
$$


then $\phi$ is a collisional invariant in the sense that

$$
\phi_{1}+\phi_{2}=\phi_{3}+\phi_{4} \quad \text { on } \mathcal{D}_{\mathrm{c}} \text {. }
$$

Under some regularity on $\omega$ and for $\int_{\mathbb{T}^{d}} d k|\phi(k)|<\infty$, it is proved in [32] that the only solutions to (B.10) are

$$
\phi(k)=a+b \omega(k) .
$$
form

If $W$ is stationary and if $\int_{\mathbb{T}^{d}} d k|\log (W /(1-W))|<\infty$, then $W$ is necessarily of the

$$
W_{\beta, \mu}^{-}(k)=\left(\mathrm{e}^{\beta(\omega(k)-\mu)}+1\right)^{-1}
$$

for some $\beta, \mu \in \mathbb{R}$. In the limit $\beta \rightarrow \infty$, one obtains

$$
W_{\infty, \mu}^{-}(k)=\mathbb{1}(\omega(k) \leq \mu)
$$

and for $\beta \rightarrow-\infty$

$$
W_{-\infty, \mu}^{-}(k)=\mathbb{1}(\omega(k) \geq \mu) .
$$

Although the corresponding collision invariant is not integrable, one checks directly that $W_{\infty, \mu}^{-}$and $W_{-\infty, \mu}^{-}$are stationary solutions of (B.1), (B.2). Presumably our list comprises all stationary solutions of the Boltzmann-Nordheim equation.

Let us introduce the density, $\rho$, and energy, e, of $W$ through

$$
\rho(W)=\int_{\mathbb{T}^{d}} d k W(k), \quad \mathrm{e}(W)=\int_{\mathbb{T}^{d}} d k \omega(k) W(k) .
$$

If one picks some $W$, then there is a unique pair $\beta, \mu$ such that $\rho(W)=\rho\left(W_{\beta, \mu}^{-}\right), \mathrm{e}(W)=$ $\mathrm{e}\left(W_{\beta, \mu}^{-}\right)$, provided one also admits the values $\beta= \pm \infty$. Because of the conservation laws, this strongly suggests that for the solution with this $W$ as initial datum it holds

$$
\lim _{t \rightarrow \infty} W(t)=W_{\beta, \mu}^{-} \text {. }
$$

On the mathematical side, besides the study of collision invariants, the main focus so far is the existence and uniqueness of solutions. We refer to the review [33]. These authors consider the momentum space $\mathbb{R}^{3}$ and dispersion $\omega(k)=k^{2}$, see Remark 5.3. To be concise we quote one result from Dobeault [34].

Theorem B.1 Let $d \geq 2$ and $\omega(k)=k^{2}$. Let $V$ be radial with $\int_{\mathbb{R}^{d}} d k|k||\widehat{V}(k)|^{2}<\infty$. For the initial datum, $W$, we assume $W \in L^{\infty}\left(\mathbb{R}^{d}\right)$ and $0 \leq W \leq 1$. Then the integrated version of the Boltzmann-Nordheim equation,

$$
W(t)=W+\int_{0}^{t} d s \mathcal{C}(W(s)),
$$

has a unique solution in $C\left([0, \infty), L^{\infty}\left(\mathbb{R}^{d}\right)\right)$, which satisfies $0 \leq W(t) \leq 1$ for all $t \geq 0$. If $\rho(W)<\infty, \mathrm{e}(W)<\infty$, and $S(W)<\infty$, then it holds, for all $t \geq 0$,

$$
\begin{aligned}
& \int_{\mathbb{R}^{d}} d k W(k, t)=\rho(W), \quad \int_{\mathbb{R}^{d}} d k k W(k, t)=\int_{\mathbb{R}^{d}} d k k W(k), \\
& \int_{\mathbb{R}^{d}} d k k^{2} W(k, t)=\mathrm{e}(W), \quad S(W(t))=S(W)+\int_{0}^{t} d s \sigma(W(s)) .
\end{aligned}
$$


Apparently, the case of interest in our context has never been investigated. One difficulty results from the constraint due to the energy $\delta$-function. On the other hand, since $\mathbb{T}^{d}$ is compact, finite number, energy, and entropy holds automatically. Under the Assumption A2 we expect Theorem B.1 still to be valid.

\section{B.2 Bosons}

The Boltzmann-Nordheim equation reads, see (5.4), (5.5),

$$
\begin{gathered}
\frac{\partial}{\partial t} W(k, t)=\mathcal{C}(W(t))(k), \\
\mathcal{C}(W)(k)=\pi \int_{\left(\mathbb{T}^{d}\right)^{3}} d k_{2} d k_{3} d k_{4} \delta\left(k_{1}+k_{2}-k_{3}-k_{4}\right) \delta\left(\omega_{1}+\omega_{2}-\omega_{3}-\omega_{4}\right) \\
\left|\widehat{V}\left(k_{2}-k_{3}\right)+\widehat{V}\left(k_{2}-k_{4}\right)\right|^{2}\left(\tilde{W}_{1} \tilde{W}_{2} W_{3} W_{4}-W_{1} W_{2} \tilde{W}_{3} \tilde{W}_{4}\right),
\end{gathered}
$$

$\tilde{W}=1+W$. It has to be solved with initial data $W$ such that $W \geq 0, \int d k W(k)<\infty$. Positivity is preserved in the course of time and number and energy conservation holds as in (B.3).

According to the Bose statistics, the entropy per volume of a quasifree state is given by

$$
S(W)=-\int_{\mathbb{T}^{d}} d k(W(k) \log W(k)-\tilde{W}(k) \log \tilde{W}(k)) .
$$

Then (B.5) to (B.11) still hold, provided $\tilde{W}=1+W$ and in (B.6) $\mid \widehat{V}\left(k_{2}-k_{3}\right)-\widehat{V}\left(k_{2}-\right.$ $\left.k_{4}\right)\left.\right|^{2}$ is substituted by $\left|\widehat{V}\left(k_{2}-k_{3}\right)+\widehat{V}\left(k_{2}-k_{4}\right)\right|^{2}$.

If $W$ is stationary and $\int_{\mathbb{T}^{d}} d k|\log W /(1+W)|<\infty$, then $W$ is necessarily of the form

$$
W_{\beta, \mu}^{+}(k)=\left(\mathrm{e}^{\beta(\omega(k)-\mu)}-1\right)^{-1},
$$

where, in order to have $W_{\beta, \mu}^{+} \geq 0$, one is restricted to

$$
\beta>0, \quad \omega_{\min } \geq \mu \quad \text { and } \beta<0, \quad \mu \geq \omega_{\max } .
$$

The density and energy of a state $W$ is still given by (B.15). Clearly, the range of this map is $D=\left\{(\rho, \mathrm{e}) \mid 0<\rho<\infty, \rho \omega_{\min }<\mathrm{e}<\rho \omega_{\max },\right\}$. However for $d \geq 3$ and under our assumptions on $\omega$, the map $(\beta, \mu) \rightarrow\left(\rho\left(W_{\beta, \mu}^{+}\right), \mathrm{e}\left(W_{\beta, \mu}^{+}\right)\right)$has a range $D_{\text {nor }}$ which is a proper subset of $D$. Therefore, for an initial $W$ with $(\rho(W), \mathrm{e}(W)) \in D \backslash D_{\text {nor }}$ the solution of the Boltzmann-Nordheim equation has no limiting stationary state. Physically the excess mass condenses to a superfluid component, which is reflected by a $\delta$-peak at $k=0$ in the Wigner function. Based on self-similar solutions it is argued that the condensate is nucleated at some finite time. We refer to the review [35], see also [36, 37].

On the mathematical side, the cubic nonlinearity of the Boltzmann-Nordheim equation poses severe difficulties. Only the case of momentum space $\mathbb{R}^{3}$, dispersion $\omega(k)=$ 
$k^{2} / 2$ has been studied in detail and even then only for isotropic solutions which makes $W(k, t)$ to depend on $|k|$ only. We use $\varepsilon$ as energy variable, $\varepsilon=k^{2} / 2$, and set $W(k, t)=$ $f(\varepsilon, t)$. In case $\widehat{V}(k)=\widehat{V}(0)=1 /\left(2(2 \pi)^{3}\right)$ the Boltzmann-Nordheim equation for the energy distribution reads

$$
\frac{\partial}{\partial t} f\left(\varepsilon_{1}, t\right)=\mathcal{C}_{\mathrm{r}}(f(t))\left(\varepsilon_{1}\right)
$$

with the collision operator

$$
\mathcal{C}_{\mathrm{r}}(f)\left(\varepsilon_{1}\right)=\int_{\left\{\varepsilon_{3}+\varepsilon_{4} \geq \varepsilon_{1}\right\}} d \varepsilon_{3} d \varepsilon_{4} \frac{1}{\sqrt{\varepsilon_{1}}} \min \left\{\sqrt{\varepsilon_{1}}, \sqrt{\varepsilon_{2}}, \sqrt{\varepsilon_{3}}, \sqrt{\varepsilon_{4}}\right\}\left(\tilde{f}_{1} \tilde{f}_{2} f_{3} f_{4}-f_{1} f_{2} \tilde{f}_{3} \tilde{f}_{4}\right),
$$

see [36]. Here $\varepsilon_{2}=\varepsilon_{3}+\varepsilon_{4}-\varepsilon_{1}, f_{j}=f\left(\varepsilon_{j}\right), j=1, \ldots, 4, \tilde{f}=1+f$. Lu [38] considers Equation (B.24) in the weak form

$$
\frac{d}{d t} \int_{0}^{\infty} \varphi(\varepsilon) f(\varepsilon, t) \sqrt{\varepsilon} d \varepsilon=\int_{0}^{\infty} \varphi(\varepsilon) \mathcal{C}_{r}(f(t))(\varepsilon) \sqrt{\varepsilon} d \varepsilon
$$

with test functions $\varphi \in C_{\mathrm{b}}^{2}\left(\mathbb{R}_{+}\right)$and proves that $(\overline{B .26})$ remains meaningful even if $f(\varepsilon, t) \sqrt{\varepsilon} d \varepsilon$ is a positive measure on $\mathbb{R}_{+}$. We denote such a measure by $f(d \varepsilon, t)$. The possible roughness of $f(d \varepsilon, t)$ is balanced by rewriting the right hand side of (B.26) in such a way that the collisional difference $\varphi\left(\varepsilon_{1}\right)+\varphi\left(\varepsilon_{2}\right)-\varphi\left(\varepsilon_{3}\right)-\varphi\left(\varepsilon_{4}\right)$ appears. For $t=0$ we assume finite mass and energy, i.e., $\int_{0}^{\infty} f(d \varepsilon)<\infty$ and $\int_{0}^{\infty} \varepsilon f(d \varepsilon)<\infty$. Then (B.26) has a solution which conserves mass and energy. The stationary measures of (B.26) are necessarily of the form

$$
f_{\beta, \mu}(d \varepsilon)=\left(\mathrm{e}^{\beta(\varepsilon-\mu)}-1\right)^{-1} \sqrt{\varepsilon} d \varepsilon+n_{\text {con }} \delta(\varepsilon) d \varepsilon
$$

with either $\mu \leq 0$ and condensate density $n_{\text {con }}=0$ or $\mu=0$ and $n_{\text {con }}>0$. The critical line dividing the normal fluid from the condensate is given by e $(\rho)=\left(\rho / \rho_{0}\right)^{-5 / 3} \mathrm{e}_{0}$ with $\rho_{0}=\int_{0}^{\infty} d \varepsilon \sqrt{\varepsilon}\left(e^{\beta \varepsilon}-1\right)^{-1}, \mathrm{e}_{0}=\int_{0}^{\infty} d \varepsilon \sqrt{\varepsilon} \varepsilon\left(e^{\beta \varepsilon}-1\right)^{-1}$.

For the particular case (B.24) one has some rigorous information on the concentration. Let the initial data be given by a density, i.e., $f(d \varepsilon)=f_{\text {ac }}(\varepsilon) \sqrt{\varepsilon} d \varepsilon$, such that

$$
\rho=\rho(f)=\int_{0}^{\infty} f_{\mathrm{ac}}(\varepsilon) \sqrt{\varepsilon} d \varepsilon<\infty, \quad \mathrm{e}=\mathrm{e}(f)=\int_{0}^{\infty} \varepsilon f_{\mathrm{ac}}(\varepsilon) \sqrt{\varepsilon} d \varepsilon<\infty .
$$

In general, the solution to (B.26) is then a measure, $f(d \varepsilon, t)$, which can be uniquely decomposed into an absolutely continuous and a singular part,

$$
f(d \varepsilon, t)=f_{\mathrm{ac}}(\varepsilon, t) \sqrt{\varepsilon} d \varepsilon+f_{\mathrm{s}}(d \varepsilon, t) .
$$

Now let $f_{\beta, \mu}$ be the equilibrium distribution corresponding to $\rho(f)$, e $(f)$, where $\mu=0$ in case $n_{\text {con }}>0$. Lu [39] proves that, for $\rho \leq \rho_{\mathrm{c}}$,

$$
\lim _{t \rightarrow \infty} \int_{0}^{\infty} f_{\mathrm{s}}(d \varepsilon, t)=0, \quad \lim _{t \rightarrow \infty} \int_{0}^{\infty}\left|f_{\mathrm{ac}}(\varepsilon, t)-f_{\beta, \mu}(\varepsilon)\right| \sqrt{\varepsilon} d \varepsilon=0 .
$$


On the other hand for $\rho>\rho_{\mathrm{c}}$ one has

$$
\lim _{t \rightarrow \infty} \int_{0}^{\infty}\left|f_{\mathrm{ac}}(\varepsilon, t)-f_{\beta, 0}(\varepsilon)\right| \varepsilon \sqrt{\varepsilon} d \varepsilon=0
$$

and

$$
\lim _{t \rightarrow \infty} \int_{\left\{\varepsilon \mid 0 \leq \varepsilon^{4} \leq r(t)\right\}} f(d \varepsilon, t)=\rho-\rho_{\mathrm{c}},
$$

where $r(t)$ is the integral in (B.31). Thus for large times the solution develops a $\delta$-peak at $\varepsilon=0$ with weight $n_{\text {con }}$.

Whether such a concentration appears after some finite time or only asymptotically, as $t \rightarrow \infty$, is left unanswered by the theorem.

\section{References}

[1] L.W. Nordheim, On the kinetic method in the new statistics and its application in the electron theory of conductivity, Proc. Roy. Soc. 119, 689-698 (1928).

[2] R.E. Peierls, Zur kinetischen Theorie der Wärmeleitung in Kristallen, Annalen Physik 3, 1055-1101 (1929).

[3] E.A. Uehling and G.E. Uhlenbeck, Transport phenomena in Einstein-Bose and Fermi-Dirac gases, Phys. Rev. 43, 552-561 (1933).

[4] L.D. Landau, The transport equation in the case of Coulomb interactions, Phys. Z. Sowj. Union 10, 154 (as no. 24 in: Collected Papers of L.D. Landau, edited by D. TerHaar, Pergamon Press, Oxford, 1965) (1936).

[5] L. van Hove, Quantum-mechanical perturbations giving rise to a statistical transport equation, Physica 21, 517-540 (1955).

[6] I. Prigogine, Nonequilibrium Statistical Mechanics, Wiley-Interscience, New York 1962.

[7] N.M. Hugenholtz, Derivation of the Boltzmann equation for a Fermi gas, J. Stat. Phys. 32, 231-254 (1983).

[8] O.E. Lanford, Time Evolution of Large Classical Systems, ed. by J. Moser, Lecture Notes in Physics 38, 1-111 (1975).

[9] E.B. Davies, Quantum Theory of Open Systems, Academic Press, London, (1976).

[10] D. Hilbert, Mathematische Probleme. In: Nachrichten der Königlichen Gesellschaft der Wissenschaften zu Göttingen, mathematisch-physikalische Klasse. Vandenhoeck \& Ruprecht, S. 253-297 (1900). 
[11] D. Robert, Semi-classical approximation in quantum mechanics. A survey of old and recent mathematical results, Helvetica Physica Acta 71, 44-116 (1998).

[12] J. Fröhlich, S. Graffi, and S. Schwarz, Mean-field and classical limit of many body Schrödinger dynamics for bosons, Comm. Math. Phys. 271, 681-697 (2007).

[13] J. Fröhlich, A. Knowles, and A. Pizzo, Atomism and quantization, J. Phys. A 40, 3033-3045 (2007).

[14] L. Erdös and B. Schlein, Quantum dynamics with mean field interaction: nonlinear Hartree equation, J. Stat. Phys., to appear.

[15] J. Lukkarinen and H. Spohn, Weakly non-linear Schrödinger equation with random initial data, in preparation.

[16] R.F. Streater, On certain nonrelativistic quantized fields, Comm. Math. Phys. 7, 9398 (1968).

[17] J. Ginibre, Some applications of functional integration in statistical mechanics, in: Statistical Mechanics and Quantum Field Theory, ed. C. DeWitt and R. Stova, pp. 327-428, Gordon and Breach, New York 1971.

[18] O. Bratteli and D.W. Robinson, Operator Algebras and Quantum Statistical Mechanics, Vol. 2, Second Edition. Text and Monographs in Physics, Springer, Berlin 1997.

[19] T.G. Ho and L.J. Landau, Fermi gas on a lattice in the van Hove limit, J. Stat. Phys. 87, 821-845 (1997).

[20] L. Erdös, M. Salmhofer, and H.T. Yau, On the quantum Boltzmann equation, J. Stat. Phys. 116, 367-380 (2004).

[21] H. Spohn, The phonon Boltzmann equation, properties and link to weakly anharmonic lattice dynamics, J. Stat. Phys. 124, 1041-1104 (2006).

[22] D.D. Botvich and V.A. Malishev, Unitary equivalence of temperature dynamics for ideal and locally perturbed Fermi gas, Comm. Math. Phys. 91, 301-312 (1983).

[23] J. Fröhlich, M. Merkli, and D. Ueltschi, Dissipative transport: thermal contacts and tunnelling junctions, Ann. Henri Poincaré 4, 897-945 (2003).

[24] L. Erdös and H.T. Yau, Linear Boltzmann equation as the weak coupling limit of a random Schrödinger equation, Comm. Pure Appl. Math. 53, 667-735 (2000).

[25] D. Benedetto, F. Castella, R. Esposito, and M. Pulvirenti, Some considerations on the derivation of the nonlinear quantum Boltzmann equation, J. Stat. Phys. 116, 381-410 (2004). 
[26] D. Benedetto, F. Castella, R. Esposito, and M. Pulvirenti, On the weak coupling limit for bosons and fermions, Math. Mod. Meth. Appl. Sci. 15, 1811-1843 (2005).

[27] D. Benedetto, F. Castella, R. Esposito, and M. Pulvirenti, From the $N$-body Schrödinger equation to the quantum Boltzmann equation: a term-by-term convergence result in the weak coupling regime, Comm. Math. Phys. 277, 1-44 (2008).

[28] V.E. Zakharov, V.S. L'vov, and G. Falkovich, Kolmogorov Spectra of Turbulence: I Wave Turbulence. Springer, Berlin 1992.

[29] T. Chen, Localization lengths and Boltzmann limit for the Anderson model at small disorders in dimension 3, J. Stat. Phys. 120, 279-337 (2005).

[30] J. Lukkarinen, Asymptotics of resolvent integrals: The suppression of crossings for analytic lattice dispersion relations, J. Math. Pures Appl. 87, 193-225 (2007).

[31] L. Erdös, M. Salmhofer, Decay of the Fourier transform of surfaces with vanishing curvature, Math. Zeitschrift 257, 261-294 (2007).

[32] H. Spohn, Collisional invariants for the phonon Boltzmann equation, J. Stat. Phys. 124, 1131-1135 (2006).

[33] M. Escobedo, S. Mischler, and M. Valle, Homogeneous Boltzmann equation in quantum relativistic kinetic theory, Elect. J. Diff. Equ. 4, 1-85 (2003).

[34] J. Dolbeaut, Kinetic models and quantum effects: a modified Boltzmann equation for Fermi-Dirac particles, Arch. Rational Mech. Anal. 127, 101-131 (1994).

[35] C. Josserand, Y. Pomeau, and S. Rica, Self-similar singularities in the kinetics of condensation, J. Low Temperature Physics 145, 231-265 (2006).

[36] D.V. Semikoz and I.I. Tkachev, Condensation of bosons in the kinetic regime, Phys. Rev. D55, 489-502 (1997).

[37] H. Spohn, Kinetics of the Bose-Einstein condensation, preprint in preparation.

[38] X.G. Lu, On isotropic distributional solutions to the Boltzmann equation for BoseEinstein particles, J. Stat. Phys. 116, 1597-1649 (2004).

[39] X.G. Lu, The Boltzmann equation for Bose-Einstein particles: velocity concentration and convergence to equilibrium, J. Stat. Phys. 119, 1027-1067 (2005). 